\title{
Euphoria, Pakikibaka, at Bahala Na: Kontekstuwalisasyon ng Piling Awiting Post-EDSA David Michael San Juan
}

Ang EDSA People Power I na nagpatalsik sa diktadurang Marcos ay nagluwal ng maraming awiting nagbibigay ng iba't ibang perspektiba hinggil sa tinatawag na "mapayapang rebolusyon." Sa gabay ng mga konsepto hinggil sa kasaysayang-bayan (people's history) nina Howard Zinn at Teresita Maceda, sinipat ng papel na ito ang pandaigdigang penomenon ng mga awiting sumasalamin sa kasaysayangbayan upang mailagay sa wastong konteksto ang paglalantad ng mga tunggalian ng mga makauring interes (class contradictions) na masisipat sa tatlong awiting post-EDSA ("Handog ng Pilipino sa Mundo," "Sayaw sa Bubog" at "Kumusta Na"). Pinaghambing din ng papel na ito ang magkakaibang pananaw ng iba't ibang uring panlipunan sa "tagumpay" at "kabiguan" ng EDSA I upang makapag-ambag sa paglalantad sa kahungkagan ng rebisyonismong historikal ng mga loyalista ng diktadurang Marcos. Sa pangkalahatan, layunin ng papel na ito na gawing aksesibol at madaling unawain para sa henerasyong post-EDSA I ang kabuluhan, kahulugan, at kontekstong pangkasaysayan ng tatlong awiting post-EDSA.

Mga Susing Salita:Awiting Filipino, EDSA, People Power, Marxismo, Kasaysayang-bayan

The EDSA People Power I that ousted the Marcos dictatorship led to the production of a number of songs giving various perspectives on the so-called "peaceful revolution." Utilizing Howard Zinn's and Teresita Maceda's concepts about people's history, this paper scrutinized the world phenomenon of songs that reflect people's history so as to contextualize class contradictions in three post-Edsa songs ("Handog ng Pilipino sa Mundo"/"The Filipinos' Offering to the World", "Sayaw sa Bubog"/"Dance on Shards" and "Kumusta Na"/"How Are You?"). This paper also compared the various perspectives of distinct social classes on the "victory" and failure of EDSA I, so as to contribute to the unmasking of the emptiness of the historical revisionism which loyalists of the Marcos dictatorship peddle. In general, the paper is aimed at making the significance, meaning and context of these three post-EDSA songs accessible and intelligible to the post-EDSA I generations.

Keywords: Filipino Songs, EDSA, People Power, Marxism, People's History

Plaridel • Vol. 16 No. 1 • January - June 2019 


\section{Panimula}

Isang pandaigdigang ekspresyon ng pakikibaka ng mga mamamayan ang paglikha ng mga awiting nagtatala ng kasaysayan ng mga kilusang panlipunan at ng matatagumpay na aklasang-bayan (People Power revolts/ uprisings). Ang mga makasaysayang awiting ito'y bahagi ng tinatawag ni Teresita Maceda (1996) ng Unibersidad ng Pilipinas na "mga tinig mula sa ibaba"-mga tinig ng ordinaryong mamamayang lumahok sa mga kilusang panlipunan at iba't ibang porma ng aklasang-bayan na nagbibigay ng alternatibong pananaw hinggil sa ilang bahagi ng kontemporanyong "piníping kasaysayan" sa gitna ng kawalan ng puwang para rito sa mga opisyal na bulwagan ng kapangyarihan. Lumaganap man o hindi, patuloy mang inawit ng iba't ibang henerasyon o agad ding nawaglit sa alaala ng mga nakibaka, ang mga awitin ng mga kilusang panlipunan at mga aklasangbayan ay makabuluhang bahagi ng "mga tinig ng kasaysayang-bayan" (voices of people's history) na "...karaniwang hindi binabanggit sa mga opisyal na bersiyon ng kasaysayan, sa mga pangunahing midya, sa mga teksbuk na madalas gamitin, sa kontroladong kultura" (Zinn \& Arnove, 2004, p. 24). Samakatwid, malaki ang pangangailangan sa pananaliksik hinggil sa mga awit ng mga kilusang panlipunan at ng mga aklasang-bayan.

Nangunguna sa talaan ng mga makasaysayang awit na bahagi ng "mga tinigmula saibaba" at "mga tinigngkasaysayang-bayan" ang "L'Internationale" [Ang Internasyunale] ng pandaigdigang kilusang sosyalista at kilusang manggagawa na isinulat ni Eugène Pottier noong 30 Hunyo 1871 bilang tugon sa anihilasyon ng Paris Commune-ang tinaguriang kauna-unahang pamahalaang pinamunuan ng mga manggagawa sa buong mundo na naging inspirasyon ng mga sumunod na sosyalista at komunista (Marx, 1871; Jiang, 2010; Mann, 2011) —at nilapatan ng musika ni Pierre Degeyter noong 1888 (Gluckstein, 2008). Bukod sa "Happy Birthday," L'Internationale" ang isa sa awiting may pinakamaraming salin sa buong daigdig. Tulad ng Bibliya, may salin sa halos lahat ng wika ang "L'Internationale," bagay na mapatutunayan sa pamamagitan ng simpleng pagsipat sa mga video ng iba't ibang bersiyon nito sa YouTube. Halimbawa, ang YouTube video ni user Tuovalla (2007) ay naglalaman ng bahagi ng mahigit 40 bersiyon ng "L'Internationale." Pamana naman ng mga partisano ng kilusang anti-pasista sa Italya noong Ikalawang Digmaang Pandaigdig ang "Bella Ciao" [Magandang Dilag, Paalam] na may bersiyon din sa iba't ibang wika gaya ng Espanyol, Turkish, Ingles, Arabic, Aleman, Mandarin, Filipino, atbp., batay sa mga video na naka-upload sa YouTube (RomanticLyrics, 2018; The Serbian Partisan, 2018; İrfan Şenozan, 2017; ي-20ody, 2018; istvan piroth, 2015; Nuprokevin, 2017; at Amoneth, 2010). Ang "Bella Ciao" ay tungkol sa isang gerilyang (partisano) antipasista na umiibig sa isang magandang dilag. Itinatagubilin niya sa iniibig 
na sakali mang mamatay siya sa pakikipaglaban, siya'y bigyan ng marangal na libing. Samantala, hanggang sa kasalukuyan ay kontrobersiyal pa rin ang "Ayesab’amagwala" o "Aw Dubul'ibhunu" ng kilusang kontra-apartheid sa Timog Africa. Panawagan ng awiting ito ang pagbaril o pagpaslang ng mga katutubong mamamayang Aprikano sa mga puting mananakop ("Boer"). Ipinagbawal ng isang hukom sa Timog Africa ang pag-awit nito dahil sa diumano'y hate speech ang ilang bahagi ng awit (Mutasa, 2011; Thompson, \& Ramhurry, 2014). Tinututulan ng ilang pinuno ng partidong African National Congress (ANC) ang pagbabawal sapagkat para sa kanila, bahagi ng paggunita sa kanilang mga pakikibaka ang pag-awit nito sa orihinal na konteksto (Dlodlo, 2011). Sa Amerika Latina, inaawit pa rin ang "El Pueblo Unido, Jamás Será Vencido" (Ortega,1975) [Ang Nagkakaisang Bayan ay Hindi Magagapi] na nilikha ng bandang Quilapayún upang ipagdiwang ang tagumpay ng mga manggagawa na nakapag-ambag nang malaki sa pagkahalal ni Salvador Allende sa Chile bilang kauna-unahang presidenteng Marxista sa buong mundo na naluklok sa pamamagitan ng eleksiyon. May bersiyon ito sa Filipino ("Awit ng Tagumpay" na inaawit ng bandang Patatag) at sa maraming iba pang wika.

Sa mga piket at strike ng mga manggagawa sa Estados Unidos at iba pang bansang gumagamit ng Ingles ay maririnig pa rin ang "Bread and Roses" at "Solidarity Forever." Isinulat ang tulang "Bread and Roses" [Tinapay at Mga Rosas] noong 1911, naging popular pagkatapos ng strike sa Lawrence, Massachussetts noong 1912 na nilahukan ng mga babaeng manggagawa o kaya'y ina o asawa ng mga manggagawa, at nilapatan ng musika noong dekada 70 (Brazill, 2009). Mula noon, naging isa na ito sa mga popular na awitin ng mga kilusang unyonista sa Amerika. Isinulat sa perspektiba ng mga ina o asawa ng mga manggagawa, panawagan ng "Bread and Roses" ang pakikibaka para sa isang makatarungang lipunan na ang karapatang magtamasa ng mga pangunahing pangangailangan ("bread") at karapatang magkaroon ng maayos, masaya, at desenteng pamumuhay ("roses") ay makakamtan na ng bawat tao.

Ginamit naman ng "Solidarity Forever" o "Pagkakaisa Magpakailanman" (1915) ang himig ng "John Brown's Body" — na siya namang pinagmulan din ng himig ng "Battle Hymn of the Republic" o "Glory, Glory Hallelujah"upang bigyan ng diin ang kahalagahan ng mga unyon sa pagtatagumpay ng kilusang manggagawa at ng kilusang sosyalista laban sa sistemang kapitalista (Gregory, 2010). Noong 2011, binuklod din ng pag-awit ng "Solidarity Forever" ang mga manggagawa sa Wisconsin dahil sa mga maniobrang kontra-unyon ni Gobernador Scott Walker (Buhle \& Buhle, 2012). Gaya ng "Bread and Roses" at "Solidarity Forever," ang "We Shall Overcome" [Tayo'y Magtatagumpay] na isinulat noong dekada 40 ay nananatiling buhay sa 
kontemporanyong kultura. Naging popular na awitin ito ng mga tumututol sa rasismo ng mga puting Amerikano laban sa mga Aprikano-Amerikano noong dekada 50 hanggang 60 (Adams, 2013). Noong Oktubre 2011, sa pagbisita ni Pete Seeger (isang popular na aktibistang folk singer sa Estados Unidos) at ng kaniyang mga kasama sa piket ng kilusang Occupy Wall Street, inawit nila ang "We Shall Overcome" ("Slumbering Colossus," 2011). Ang kilusang Occupy Wall Street ay nagsimula bilang isang lokal na protesta sa Amerika laban sa pagiging ganid ng mayayamang kapitalista na bumubuo sa top 1\% ng populasyon ng Estados Unidos. Ayon sa occupywallst.org (ang pinakauna at pinakapopular na website ng kilusang Occupy Wall Street/ OWS), "ang kilusan ay inspirado ng mga popular na aklasan sa Ehipto at Tunisia at lumalaban sa pinakamayamang $1 \%$ ng populasyon na nagtatakda ng mga tuntunin ng isang di makatarungang ekonomyang global..." (para. 1) Sa kasalukuyan, may bersiyon na ang kilusang OWS sa halos bawat pangunahing lungsod at sentrong bayan sa buong mundo.

Ang bansang Pilipinas ay may mga awiting sumasalamin din sa kasaysayang-bayan gaya ng tulang "Bayan Ko" na isinulat ni Jose Corazon "Huseng Batute" de Jesus noong dekada 20 nilapatan ng musika ni Constancio de Guzman upang tuligsain ang imperyalismong Amerikano na umagaw sa kalayaan ng kasisilang pa lamang na Republika ng Pilipinas, ngunit higit na naging popular bilang awitin ng protesta sa panahon ng Batas Militar na ipinataw ng diktadurang Marcos mula 1972 hanggang 1981. Batay sa pananaliksik ni Maceda (1996), maraming awiting bahagi ng kasaysayang-bayan ang isinulat ng mga kasapi ng lumang Partido Komunista ng Pilipinas o PKP (na itinatag noong 1930 kaya tinatawag ding PKP-1930) at ng Partido Sosyalista ng Pilipinas, na halos kapanahon ng pagiging awit ng tulang "Bayan Ko." Batay sa mga sangguniang online gaya ng Pambansa-Demokratikong Paaralan o Padepa-na pinamamahalaan ng Pambansang Kagawaran ng Edukasyon ng bagong Partido Komunista ng Pilipinas (higit na kilala bilang Communist Party of the Philippines o CPP)— aktibo rin ang bagong Partido Komunista ng Pilipinas sa paglikha ng mga awiting sumasalamin sa kasaysayang-bayan, partikular sa perspektiba ng kanilang armadong rebolusyon na nagsimula noong 1969 sa pamamagitan ng pagtatatag ng Bagong Hukbong Bayan o New People's Army (NPA) ang opisyal na hukbo ng CPP. Matatagpuan sa http://www.padepaonline. com/index.php/awit ang archive ng ilang awit na nilikha ng mga grupong pangkultura ng CPP. May sarili ring archive ng "mga awit ng Rebolusyong Pilipino" ang Filipino Artists for National Democracy na matatagpuan naman sa link na ito: http://revsongs.tripod.com/. Batay sa ginawang pagbabasa-basa ng mananaliksik, wala pang komprehensibong pag-aaral na kahawig ng ginawa ni Maceda para sa mga awitin ng PKP-1930 at ng 
Partido Sosyalista ng Pilipinas, ang naisabalikat para sa mga awitin ng CPP. Gayunman, noong 1998 ay inilathala ng University of the Philippines Press ang "Muog: Ang Naratibo ng Kanayunan sa Matagalang Digmang Bayan sa Pilipinas" na pinamatnugutan ni Gelacio Guillermo at inihanda ng Instityut sa Panitikan at Sining ng Sambayanan (IPASA). Ang "Muog" ay "kalipunan ng mga liham, talakayambuhay, talaarawan, reportahe, panayam, parangal/ paggunita, pabula, dagli, sugilanon ("kuwento" sa Bisaya), maikling kwento, at bahagi ng nobela" (Guillermo, 1998) ng mga kadre (pinuno) at masang pinamumunuan ng CPP na sumasaklaw sa panahong mula 1972 hanggang 1997.

Ang mga awiting bahagi ng "mga tinig mula sa ibaba" at "mga tinig ng kasaysayang bayan" ay karaniwang sumasalamin sa mga tunggalian ng mga uri (class contradictions) sa lipunan, lantad man o hindi. Narito ang isang talahanayan na nagpapakita sa tunggalian ng mga uri na umiiral sa mga nabanggit na awitin ng mga kilusang panlipunan at mga aklasang-bayan:

Table 1. Tunggalian ng mga uri sa piling awit ng mga kilusang panlipunan.

\begin{tabular}{|l|l|}
\hline \multicolumn{1}{|c|}{ AWIT } & \multicolumn{1}{c|}{ TUNGGALIAN } \\
\hline "L'Internationale" & Uring Manggagawa vs. Uring Kapitalista \\
\hline "Bella Ciao" & Partisanong Anti-Pasista vs. Kapitalistang Pasista \\
\hline "Ayesab'amagwala'" & Katutubong Aprikano vs. Puting Mananakop \\
\hline "Solidarity Forever" & Uring Manggagawa vs. Uring Kapitalista \\
\hline "Bread and Roses" & Uring Manggagawa vs. Uring Kapitalista \\
\hline "We Shall Overcome" & $\begin{array}{l}\text { Puwersang Anti-Rasista (karamiha'y Aprikano- } \\
\text { Amerikano) vs. Puwersang Rasista (karamiha'y } \\
\text { Amerikanong puti) }\end{array}$ \\
\hline "Bayan Ko" & $\begin{array}{l}\text { Katutubong Populasyon vs. Dayuhang } \\
\text { Kolonyalista }\end{array}$ \\
\hline "El pueblo unido, jamás será vencido" & Mga Manggagawa vs. Mga Kapitalista \\
\hline
\end{tabular}

\section{Metodolohiya}

Ang tagumpay ng Edsa People Power I na nagpatalsik sa diktadurang Marcos ay nagluwal din ng maraming awiting bahagi ng kasaysayangbayan na nagbibigay ng iba't ibang perspektiba hinggil sa tinatawag na "mapayapang rebolusyon" gaya ng "Handog ng Pilipino sa Mundo" ni Jim Paredes, "Sayaw sa Bubog" ng The Jerks at "Kumusta Na" ng Yano. Isasailalim ng papel na ito ang nabanggit na tatlong awiting post-Edsa sa proseso ng Marxistang kontekstuwalisasyon upang mailantad ang mga tunggalian ng mga makauring interes (class contradictions) na namamayani sa bawat awit ng kasaysayang-bayan. Pinili ng mananaliksik ang tatlong awiting ito dahil sa naobserbahang pagkakaiba-iba ng perspektiba ng mga 
ito. Bukod dito, wala pang makabuluhang entri sa Google Scholar (online database ng mga pananaliksik) hinggil sa "Sayaw sa Bubog" at "Kumusta $\mathrm{Na}$ " ng Yano. Bagama't may ilang pananaliksik na ang isinagawa hinggil sa "Handog ng Pilipino sa Mundo" at tinalakay na rin ito sa aklat na Musical Renderings of the Philippine Nation ni Christi-Anne Castro (2011), wala sa mga nabanggit na pag-aaral ang gumamit ng lenteng Marxista sa pagsipat ng mga nabanggit na awitin.

Wala pa ring tesis o disertasyon sa mga nangungunang unibersidad sa Metro Manila na gumamit ng Marxistang kontekstuwalisasyon sa paghahambing ng tatlong awiting ito na may eksposyur na rin naman sa midyang mainstream. Halimbawa, isang bersiyon ng "Handog ng Pilipino sa Mundo" ang inawit nang live sa misa bago ilibing ang yumaong dating Pangulong Corazon C. Aquino (Tan, 2009). Ang "Kumusta Na" ay ginamit namang background sound ng ABS-CBN 2 sa isang programang nagsusuri sa kalagayang pambansa sa panahong malapit na ang pagbasa ng pangulo sa State of the Nation Address noong 2011. Taong 1994 nang inilabas ang "Kumusta Na" kasama ng ibang mga kanta sa isang album na Yano. Mabilis na naging hit album at ikinategoryang "platinum record" (Floro, 2017) Naging popular din sa radyo ang awiting ito ng Yano noong dekada 90. Samantala, pinatugtog naman ang entradang instrumental ng "Sayaw sa Bubog" sa isang edisyon ng Pinoy Big Brother. Sa kasamaang-palad, ang eksposyur ng tatlong awiting ito sa midyang mainstream ay paimbabaw lamang o superpisyal dahil ang "Handog ng Pilipino sa Mundo," "Kumusta $\mathrm{Na}$ " at "Sayaw sa Bubog" ay "itinatanghal" (made visible) "ngunit ang kaligirang panlipunan at pampulitika sa maraming kaso ay inaalis" ["but the social and political background in most cases is cut out"] (Gržinić, 2011, p. 27), kung ilalapat sa Pilipinas ang kahawig na obserbasyon ni hinggil sa mga likhang-sining na mula sa dating Blokeng Silanganin/former Eastern Bloc (mga bansa sa Silangang Europa na dating impluwensyado ng Unyong Sobyet o Union of Soviet Socialist Republics/USSR). Sa kaso ng "Handog ng Pilipino sa Mundo," bagama't literal na nakaukit sa pader ng Our Lady of Peace Shrine sa EDSA ang ilang bahagi nito, ay wala namang pagkakataon para sa aktuwal na popularisasyon nito. Samakatwid, makapag-aambag nang malaki ang Marxistang kontekstuwalisasyon upang mailigtas ang mga makasaysayang awit na ito sa napipintong paghantong sa kawalangkabuluhan sa pananaw ng mga mamamayan at pagkawaglit sa kolektibong kamalayan.

Ang kontekstuwalisasyon ay tumutukoy sa paglulugar ng isang partikular na akdang pampanitikan gaya ng tula, nobela o awit, sa panahong pinagmulan nito. Sa prosesong ito'y inilalarawan ang lipunang nagluwal sa akdang pampanitikan, partikular ang mga kondisyong sosyo-ekonomiko ng 
mga mamamayan sa panahong iyon. Alinsunod ito sa pananaw ni Antonio Gramsci at ng iba pang Marxista at realista na hindi maaaring ihiwalay ang likhang-sining at ang indibidwal na lumikha nito, sa lipunang kaniyang pinagmulan: Ang alagad ng sining ay hindi lamang nagsusulat o nagpipintakumbaga, hindi niya binibigyang-buhay ang kaniyang imahinasyon-para lamang sa kaniyang sariling pag-alaala, para lamang kaniyang sariwain ang oras ng paglikha. Siya'y nagiging alagad ng sining lamang sa pamamagitan ng kongkretisasyon, obhetisasyon, at historisisasyon ng kaniyang mga imahinasyon (Gramsci, 1985, p. 112, salin ng may-akda).

Batay sa mga ipinahayag ni Gramsci (1985), kung pahihintulatan ang paglalaro ng mga salita, ang imahinasyon ng isang alagad ng sining ay hindi simpleng imahinasyon kundi isang manipestasyon ng imahen ng kaniyang nasyon o lipunang ginagalawan. Interesado ang mga Marxista sa kontekstuwalisasyon ng mga akdang pampanitikan gaya ng mga tula at awit dahil sa mapagmulat at rebolusyonaryong potensiyal ng mga ito gaya ng pinatutunayan ng patuloy na pamamayagpag ng mga awitin ng mga kilusang panlipunan at aklasang-bayan sa kasaysayan ng daigdig.

Sa kanilang kapanahunan, ang mga dakilang guro ng kilusang sosyalista at komunista (o Marxista) na sina Karl Marx at Friedrich Engels ay kapuwa nagsulat ng mga artikulong gumagamit ng kontekstuwalisasyon ng mga akdang pampanitikan, bagama't ang mga akdang ito'y di kasingpopular ng kanilang mga sulating politikal gaya ng Communist Manifesto" at Das Kapital. Halimbawa, sa "Critical Notes on the Article: The King of Prussia and Social Reform By a Prussian" (1844), nilinaw ni Marx ang mensaheng anti-kapitalista ng tulang "Die Schlesischen Weber" o "Weaver's Song" ("Awit ng Maghahabi”) ni Heinrich Heine. Paksa ng tula ni Heine ang rebelyon ng mga manggagawang Silesian noong 1844. Si Friedrich Engels ang kaunaunahang nagsalin sa Ingles ng "Die Schlesischen Weber" (Ashton, 2011). Isinalin din ni Engels (1865) ang "Herr Tidmann" [Ginoong Tidmann], isang awiting-bayan o folk song mula Denmark na pumapaksa sa pagpatay ng isang matandang magsasaka kay Ginoong Tidmann, isang mapang-aping panginoong maylupa (landlord). Sa isang sulat naman noong 7 Pebrero 1856, ibinalita ni Engels kay Marx ang tungkol sa isang "munting awitin" ng mga manggagawa sa Paris na tumutuligsa kay Napoleon Bonaparte. Ayon kay Engels, ang popularidad ng nasabing awitin ay ebidensiya ng napipintong paghina ng kapangyarihan ni Bonaparte. Sa isa namang liham kay Hermann Schlüter noong 15 Mayo 1885, inisa-isa ni Engels ang mga rebolusyunaryong awitin sa kaniyang panahon (kabilang na ang "Herr Tidmann"). Ayon sa nasabing liham, inilathala ni Engels sa Der Sozialdemokrat ang salin ng "Herr Tidmann" noong 1865. Malinaw sa mga halimbawang ito na mismong sina 
Marx at Engels ay nagbigay-diin sa kahalagahan ng kontekstuwalisasyon ng mga iba't ibang porma ng midya.

Bukod sa paglalantad sa mga tunggaliang panlipunan sa tatlong awiting post-EDSA, paghahambingin din ng papel na ito ang magkakaibang pananaw ng iba't ibang uring panlipunan sa "tagumpay" at "kabiguan" ng EDSA I upang makapag-ambag sa paglalantad sa kahungkagan at kahunghangan ng rebisyonismong historikal ng mga loyalista ng diktadurang Marcos. Bibigyang-diin sa papel na ito ang naobserbahang pag-iral ng tatlong namamayaning bersiyon ng kasaysayang post-EDSA I: euphoria sa diumano'y "nanumbalik" na demokrasya, panawagan ng patuloy na pakikibaka bunsod ng pagpapatuloy ng mga patakarang sosyo-ekonomiko ng pinatalsik na diktadura sa panahon ng "bagong demokrasya," at ang tipikal na pag-uugaling "bahala na" na sumasagisag sa pagpapatuloy ng nakagisnang buhay, may pakikibaka man o wala, may diktadura man o wala. Inaasahang magiging aksesibol at madaling unawain para sa henerasyong post-EDSA I ang kabuluhan, kahulugan at kontekstong pangkasaysayan ng tatlong awiting post-EDSA sa pamamagitan ng pag-aaral na ito. Modelo ng pananaliksik na ito ang inilimbag na pananaliksik ni Maceda na pinamagatang Mga Tinig Mula sa Ibaba: Kasaysayan ng Partido Komunista ng Pilipinas at Partido Sosialista ng Pilipinas sa Awit, 1930-1955 (1996), ang aklat nina Howard Zinn at Anthony Arnove na Voices of a People's History of the United States (2004), at ang aklat ni Robbie Lieberman na My Song Is My Weapon: People's Songs, American Communism, and The Politics of Culture, 1930-1950 (1995). Lohikal na pagpapatuloy rin ito ng papel na "Ang Bagong Lipunan, 1972-1986: Isang Panimulang Pag-aaral sa Musika at Lipunan” ni Raul Navarro ng Unibersidad ng Pilipinas (2008).

\section{Kuwento sa Likod ng mga Awit: \\ Ang mga May-akda at ang Kanilang "Politika”}

Ang "Handog ng Pilipino sa Mundo" ay nilikha ni Jim Paredes (1986). Si Jim Paredes ay bahagi ng bandang Apo Hiking Society (APO). Apolinario Mabini Hiking Society ang orihinal na pangalan ng pangkat na nang magsimula'y may mahigit 10 miyembro (Lolarga, 2002). Graduate ng Ateneo de Manila University ang tatlong nagtagal na miyembro ng Apo-si Jim Paredes, Buboy Garovillo, at Danny Javier. Sa aspektong komersyal, mula noong dekada 70 hanggang 80 (Lockard, 1998)at sa patuloy na popularidad ng kanilang mga sikat na awitin, hindi kalabisang sabihing matagumpay ang mainstream singing career ng Apo. Ang concert nila na pinamagatang "APO KayangKaya Pa," noong 2009 ay may price range na 1,000 hanggang 2,000 piso (Orosa, 2009). Ayon mismo kay Javier, sa panahon ng diktadurang Marcos, may mga concert sila sa mga hotel ballroom na ang price range ay 1,200 
hanggang 2,500 piso (Lolarga, 2002). Ang popularidad ng kanilang mga awitin ay pinatunayan ng paglalabas noong 2006 ng Kami nAPO Muna, isang all-star tribute album na naglalaman ng cover ng mga sikat na kanta ng Apo. Kung isasaalang-alang ang matagumpay nilang mainstream singing career, masasabing kabilang sila sa upper segment ng petiburgesya (o mas mataas pa).

Walang pakikisangkot sa anumang organisasyong aktibista ang Apo bilang isang pangkat, bagama't aktibo itong tumulong sa propagandang anti-Marcos sa pamamagitan ng mga satirikal na konsiyerto pagkatapos ng asasinasyon sa isa sa mga lider ng oposisyon na si Sen. Benigno "Ninoy" Aquino, Jr. (Caparas, 2004). Lumahok din sa mga kilos-protestang antiMarcos at sa Edsa People Power I ang tatlong kasapi ng Apo (Lolarga, 2002; Ching, 2011). Si Javier ay isa rin sa mga pangunahing convenor ng Kongreso ng Mamamayang Pilipino II o Kompil II (Tubeza, 2001). Ang Kompil II ay isang organisasyong multisektoral na itinayo sa layuning pagkaisahin ang mga indibidwal at pangkat na nananawagan ng pagbibitiw o pagpapatalsik sa puwesto ng noo'y Pangulong Joseph Ejercito Estrada (ang Kompil I ay binuo naman sa panahon ng diktadurang Marcos). Instrumental ang Kompil II sa tagumpay ng EDSA Dos/II na nagpatalsik kay Estrada noong 2001 (Velasco, 2004). Lumahok sa Edsa II sina Paredes at Javier (Caparas, 2004). Batay sa kaniyang mga pahayag sa publiko, si Paredes ay isa ring aktibistang antiGloria Macapagal-Arroyo (Paredes, 2007). Noong eleksyong presidensyal ng 2010, pormal na ipinahayag ng Apo ang kanilang pagsuporta sa kandidatura ng noo'y Senador Benigno Simeon "Noynoy" C. Aquino III sa pamamagitan ng kanilang kahuli-hulihang concert bilang grupo na isinagawa noong Mayo 2010 sa Music Museum sa Greenhills (Malalad, 2010). Tinuldukan nina Javier, Paredes at Garovillo ang apat na dekadang pag-awit ng Apo bilang grupo sa araw na iyon. Sa kasalukuyan, aktibong kritiko ng administrasyong Duterte si Paredes, batay sa kaniyang mga regular na post sa social media.

Sa pagrerekord ng nasabing awit, pinili ng APO ang iba pang mangaawit na may paninindigang anti-diktadura katulad ng sa kanila:

We chose artists who had gone out of their comfort zones and spoken out against the regime at some point. The cast consisted of the APO, Celeste Legaspi, Noel Trinidad, Subas Herrero, Coritha \& Eric, Edru Abraham, Ivy Violan, Inang Laya (Karina David and Becky Demetillo), Joseph Olfindo, Kuh Ledesma, Lester Demetillo, and Leah Navarro. (Paredes, 2016, para. 11)

Ang ilan sa mga mang-aawit na ito ay nagpatuloy sa kani-kanilang politikal na pakikisangkot sa iba't ibang panahon. Halimbawa, ang Inang 
Laya ay patuloy na lumikha ng mga awitin ng protesta na hanggang ngayo'y popular pa rin sa sirkulo ng mga aktibista gaya ng "Titser" (awit na popular sa mga gurong aktibista sa mga paaralang publiko dahil sa malinaw na paglalarawan ng abang kalagayan ng mga guro at ng bayan at ng hamon sa mga guro na makilahok sa "pambansang pagbabago") at "Babae," (hindi natatapos na awit ayon kay Andrada (2016). Si Herrero, gaya ng APO ay nanawagan din ng pagbibitiw sa puwesto ni Joseph Estrada sa panahon ng Edsa Dos (Llanes, 2013; Sulat, 2013). Samantala, si Noel Trinidad naman ay nakilahok sa protesta ng mga mamamayan laban sa pork barrel sa panahon ng ikalawang administrasyong Aquino ("Thousands join million people march vs. pork," 2013), at petitioner din laban sa paglilibing ng diktador na si Marcos sa Libingan ng mga Bayani (Lopez, 2016). Personal ring nakita ng mananaliksik si Trinidad sa isang malaking kilos-protesta laban sa charter change sa mga huling taon ng rehimeng Macapagal-Arroyo. Si Navarro ay aktibong kalahok din sa EDSA Dos at sa mga kilos-protesta laban sa rehimeng Macapagal-Arroyo (Jimenez, 2008). Kritiko rin siya ng administrasyong Duterte batay sa kaniyang mga komento sa social media at sa aktwal na komprontasyon sa mga tagasuporta ng rehimen (Severo, 2018; Bantayan, 2017; "Tensions erupt between Duterte Youth and Jim Paredes at EDSA 31 celebration," 2017).

Samantala, mga aktibista naman ang bumubuo ng The Jerks, ang bandang lumikha sa awiting "Sayaw sa Bubog." Aktibo sa kilusang antiGloria ang The Jerks. Ang bokalista at pangunahing gitarista ng banda na si Chikoy Pura kasama ang ilan pang miyembro ng The Jerks ay tumutugtog pa rin hanggang ngayon sa mga kilos-protesta ng mga aktibistang grupo, batay sa mga balita sa pahayagan at sa aktuwal na pagsaksi ng mananaliksik. Pinatutunayan ito ng mga video ng kanilang pagtatanghal sa mga nasabing protesta na makikita sa www.arkibongbayan.org. Tahas na politikal ang nilalaman ng karamihan sa iba pang awit ng The Jerks.

Nilikha naman ng bandang Yano ang "Kumusta Na." Si Dong Abay ang bokalista at lirisista ng Yano na ngayo'y disbanded na. Si Abay ay graduate ng Unibersidad ng Pilipinas na isa sa mga paaralang itinuturing na balwarte ng aktibismo. Ayon sa ngayo'y defunct na na Multiply site ng bandang Patatag, si Abay at ang isa pa sa tatlong orihinal na miyembro ng Yano na si Eric Gancio ay mga dating kasapi ng Patatag (Valeros, 2006), isang progresibong banda na kilala dahil sa kanilang mga awiting tahas na politikal na lumaganap simula noong mga huling taon ng diktadurang Marcos. Maraming awitin ng Yano ang may mensaheng panlipunan at pampolitika gaya ng "Trapo," "Esem" at "McJo." Bukod dito, gaya ni Chikoy Pura, bahagi rin ng kilusang antiGloria si Dong Abay. Kapuwa rin sila kritiko ng ikalawang administrasyong Aquino at maging ng administrasyong Duterte (Artists for the Removal of 
Gloria, 2006; De Lara, 2008; “Walang Noranian na naging drug addict dahil kay Nora': Fans rail vs PNoy's reason for National Artist snub," 2014; Fans rail vs PNoy's reason for National Artist snub," 2014; Ayroso, 2015; kodao phils, 2015; Olea, 2018; Caruncho, 2018).

Sa kabuuan, may direktang koneksiyon sa mga ordinaryong mamamayan ang Apo at ang ilan pa sa mga bahagi ng pangkat na umawit ng "Handog ng Pilipino sa Mundo," The Jerks at Yano dahil sa kanilang paglahok sa mga kilos-protesta, pagtugtog sa mga kilos-protesta at paglikha ng awiting sumasalamin sa mga pakikibaka ng mamamayan. Gayunman, sa tatlong banda, higit na lantad at malinaw ang koneksiyon ng The Jerks sa masa dahil sa patuloy nitong paglahok sa mga kontemporanyong kilos-protesta ng mga aktibistang grupo. Ang ganitong koneksiyon (o ang kakulangan nito) ang humubog sa pagkakaiba-iba ng perspektiba ng tatlong banda sa pag-iral ng tunggalian ng mga uring panlipunan, at sa kinahinatnan ng EDSA I.

\section{Tunggalian ng Mga Uri:}

\section{Sa Ilalim ng Karpeta, sa Ibabaw ng Mesa at sa Kalsada}

Sa "Handog ng Pilipino sa Mundo" ng Apo na isinulat sa unang panauhan o first person (gumagamit ng "ko") tila isinantabi ang pag-iral ng tunggalian ng mga uri sa lipunang Filipino alang-alang sa pagkakaisa ng mga puwersang kontra-diktadura na "di na" "papayag" na "mawala" pang muli o kaya'y "mabawi" ang "kalayaan" na "kaytagal nating mithi." Para sa Apo, ang "mapayapang paraang pagbabago" ay makakamtan "basta't magkaisa tayong lahat." Sa pananaw ng Apo, ang EDSA ay isang aklasang supraclass, isang pag-aalsang anti-diktadura lamang, walang uring namamayani o nagpapatakbo ng mga pangyayari, ang kaaway ay ang diktadura lamang: "Masdan ang nagaganap sa aming bayan/Magkasama nang mahirap at mayaman/Kapit-bisig madre, pari, at sundalo/Naging Langit itong bahagi ng mundo" (Paredes, 1986). Problematiko ang gayong pagbibigay-diin sa espontanyong pagkakaisa laban sa diktadura kung isasaalang-alang ang katotohanan na sa mahabang panahon bago mag-EDSA Uno ay matapat at mabalasik na tagapagpatupad ng Batas Militar ang halos buong aparatong pulis-militar ng bansa (Hernandez, 1985). Sa aktuwal, ang bilis ng oportunistikong pagbaligtad ng aparatong pulis-militar ay kahawig ng bilis ng pagbagligtad ng gobyerno ng Estados Unidos na sa kabila ng mga pangaabuso sa karapatang pantao sa ilalim ng diktadurang Marcos ay patuloy na nagbigay ng malaki at malawak na ayudang militar dito (Hawes, 1986).

Samakatwid, tila ipinailalim ng Apo sa karpeta o carpet ("swept under the rug," sabi nga sa "Where is the Love" ng Black-Eyed Peas (Adams et al., 2003) ng panawagang demokrasya laban sa diktadura ang pag-iral ng tunggalian ng mga uri (magsasaka at maralitang tagalungsod versus panginoong maylupa/ 
landlord at/o landgrabber; manggagawa versus kapitalista; sundalong mersenaryo ng diktadura versus mga aktibistang anti-diktadura). Dapat bigyang-diin na ang diktadurang Marcos ay isang rehimeng tahas na naging kakampi ng mga panginoong maylupa, kapitalista atbp. kaya naman tinawag ni Anderson (1988) si Marcos na "supreme cacique." Ang "cacique" ay terminong nangangahulugang "panginoon," "boss" o "amo." Bilang supreme cacique, walang ginawa ang diktadurang Marcos kundi ipagpatuloy ang mga patakarangsosyo-ekonomikong mga nagdaang administrasyon na walangiba kundi pag-akit sa dayuhang pamumuhunan (foreign investment), pagkatali sa agrikultura, kawalan ng industriyalisasyon, pagpapanatili ng kawalan ng lupa (landlessness) ng mga magsasaka at pagpapalala ng kahirapan ng mga ordinaryong mamamayan habang nagbibigay ng ilang konsesyon sa kapuwa elite (Litonjua, 2001; Aquino, 1987). Makabuluhang sipiin sa orihinal ang obserbasyon ng Amerikanong ekonomista na si Boyce (1993) hinggil sa sitwasyon ng Pilipinas sa panahon ng diktadurang Marcos:

\begin{abstract}
Although per capita income in the Philippines rose between the early 1960s and the mid-1980s, the incomes of the country's poor majority declined. Real wages fell sharply in both rural and urban areas, even in periods when the country was experiencing relatively rapid growth in national income...Equity was not 'traded off' for growth in the Philippines. Rather, both were sacrificed to a technocratic development strategy wedded to an unjust political and economic order. (p. 4)
\end{abstract}

Nilinaw rin ni Boyce (1993) na hungkag [empty] at maka-status quo ang programang pang-ekonomya ng diktadurang Marcos, bagay na lalong nagpapatibay sa kaniyang gampanin bilang supreme cacique: "The technocrats who formulated Philippine development strategy under President Marcos did not challenge the country's inegalitarian economic and political order" (p. 8). Hindi nakinabang ang mga ordinaryong mamamayan sa mga sinasabing "benepisyo" sa ilalim ng diktadurang Marcos kaya hindi kataka-takang ang tunggaliang mayaman versus mahirap, kapitalista versus manggagawa, atbp. ay umiral sa panahong iyon sa anyo ng mga kilos-protesta, piket at strike ng mga manggagawa, maralitang tagalungsod (urban poor) at mga magsasaka sa pangunguna ng mga puwersa sa Kaliwa (Fagan, 2008; Youngblood, 1978; Kimuell-Gabriel, 2013). Isang ebidensiya ng pag-iral ng tunggalian ng mga uri sa panahon ng diktadura ang pagbabawal ni Marcos sa mga strike ng mga manggagawa at iba pang uri ng protesta nang ideklara ang Batas Militar (Wurfel, 1977). Sa kabila ng opisyal na pagbabawal, maraming demonstrasyon ng mga manggagawa para sa mas mataas na 
sahod, ng mga magsasaka para sa reporma sa lupa, ng mga katutubo laban sa pangangamkam ng kanilang mga lupain, at ng mga aktibistang nagtataguyod ng karapatang pantao laban sa mga pang-aabuso ng diktadura ang naitala sa ilalim ng rehimeng Marcos (Roces, 2004).

Sa panahon ng diktadurang Marcos ay nagsimulang lumakas ang kilusang komunista na binubuo ng Communist Party of the Philippines (CPP) at ng hukbo nito na tinatawag na New People's Army (NPA). Ayon kay Steinberg (1987):

Marcos who had cited the communist threat as the justification for Martial Law, was the best recruiter the NPA could have had. His mismanagement of the economy, his greed and the greed of his associates, and the growing contradictions of Philippine society drew the hungry, homeless, and hopeless to the NPA. (p. 237)

Naakit ang maraming mamamayan sa CPP at NPA dahil sa pagbibigay nito ng alternatibo sa di makatarungang sistema sa ilalim ng diktadura na pumapabor sa ilang sektor lamang ng elite, isang sistema na tinaguriang "crony capitalism" (Abinales \& Amoroso, 2005).

Dahil nagpokus ang "Handog ng Pilipino sa Mundo" sa supraclass na rurok ng EDSA Uno, wala itong espasyo at panahon para sa paggunita sa hindi kumbinyenteng katotohanan na ang pinakamayayaman sa bansa (Tiglao, 2015) ay nanatiling kasabwat (o "kritikal na kolaboreytor") ng diktadura hanggang sa maging malawak na malawak na ang protestang anti-diktadura ng masang Filipino, bagay na direktang kabaligtaran ng maaga at matibay na paninindigang anti-diktadura ng masang kalinya ng Kaliwa sa bansa. Katunayan, ang asasinasyon lamang ni Senador Benigno "Ninoy" Aquino, Jr. ang naging titis o spark ng malawakang pag-abandona ng panggitnang uri sa kalunsuran (Mydans, 1986) at elite sa diktadurang Marcos (Abinales \& Amoroso, 2005). Sa ganitong konteksto, kapuna-puna ang pagsasantabi ng "Handog ng Pilipino sa Mundo" sa tunggalian ng mga uri-na tumatagos sa magkakaibang proyektong politikal: isang repormistang konstitusyunal at isang radikal na suprakonstitusyonal-na malinaw na umiral sa ilalim ng rehimeng Marcos at umiiral pa rin sa ilalim ng mga administrasyong postEDSA (Docena, 2006; Ylagan, 2014).

Sa pangkalahatan, ipinalalaganap ng "Handog ng Pilipino sa Mundo" ang pananaw na may pagka-utopiko [utopian] kaugnay ng euphoria sa pagbagsak ng diktadura. Pagdiriwang sa pagkakaisa ng buong bayan ang pangunahing ikinikintal ng awit na ito, bunga na rin ng konteksto ng paglikha ni Paredes (2016) sa awiting ito na ayon mismo sa kaniya ay sarili niyang paglalarawan sa "...unique moment that inspired many democratic movements in the 
world..." (para. 19) na sa pormang music video nito ay inasahan niyang maging isang "experience that can unite and inspire a nation" (para. 19). Kalinya ito ng kampong liberal-demokratiko sa politika na nakapokus lamang sa kalayaang sibil at politikal, at hindi nangangahas lumagpas sa panawagang demokrasya at mabuting pamamahala o good governance (Rivera, 2001) na inaakalang mapagtutulungan ng mayayaman at mahihirap (gaya ng sigabo ng EDSA Uno), kaiba sa radikal na Marxistang proyekto na naghahangad din ng radikal na pagbabago sa sistemang ekonomiko tungo sa isang lipunang wala nang mayaman at mahirap.

Ang limitasyon ng perspektiba ng "Handog ng Pilipino sa Mundo" ay makikita sa pagiging "poster boy" mismo ng EDSA Uno sa mata ng mga tinaguriang mapayapang rebolusyong demokratiko na binanggit din ni Paredes (2016): "fall of the Berlin Wall, the break-up of the Soviet Union, and the student uprising in China's Tiananmen Square" (para. 19). Sinasalamin ng perspektiba ni Paredes ang paggamit ng mga larawan sa mga aklasan sa mga bansang komunista para sa isang music video ng bersiyong Ingles ng awiting ito na pinamagatang "A New And Better Way (The People's Anthem)" na ini-upload sa YouTube ni Cabling (2013). Kung gayon, ideolohiko ang pagbibigay-pokus ni Paredes sa "mapayapang paraang pagbabago." Ang gloripikasyon ng EDSA Uno ay tila malay na itinaguyod ni Paredes bilang pangontra sa ideya ng armadong rebolusyon (na maagang bahagi pa ng diktadura ay sumusulong na) at/o sa komunismo, bagama't kakatwang sa unang minuto ng music video nito na nilikha ni Mike De Leon (Paredes, 2007) ay binibigyang-pugay ang mga Katipunero, at si Andres Bonifacio mismo (na kapuwa itinuturing na inspirasyon ng mga Filipinong komunista). May pangangailangang basagin ang umiiral na mito na ang supraclass na rurok ng mga aklasang bayan ang tanging nagpabagsak sa diktadurang Marcos. Sa konteksto ng malawak na ambag ng Communist Party of the Philippines (CPP) sa pakikibakang anti-Marcos, bukod pa sa katotohanang binigyangdaan ng politikal na pag-oorganisa ng Kaliwa ang People Power (Curaming \& Claudio, 2010), dapat bigyan ng diin na ang EDSA Uno ay kulminasyon lamang ng mga kilos-protesta at pakikibaka ng mga ordinaryong mamamayan na ang iba ay simpatetiko rin sa armadong pakikibaka (gaya ng pinatutunayan ng lakas at saklaw ng CPP sa mga panahong iyon), gaya ng paggunita ni Remollino (2006) na nagtala ng 20 mahahalagang personahe (karamihan ay nasa kampong Kaliwa o nakakiling sa Kaliwa) na nakibaka sa diktadurang Marcos "mula simula," mga personaheng ang pagsisikhay ay naghasik ng binhi ng, dumulo, o kaya'y nakapag-ambag kahit paano sa pagtatagumpay ng EDSA Uno. Karamihan sa 20 personaheng tinukoy ni Remollino ay pawang kakampi ng mga tinatawag na pinagsasamantalahang uri sa marami-raming isyu at adbokasiyang ekonomiko, bagay na hindi 
sinaklaw ng mensahe ng "Handog ng Pilipino sa Mundo." Sa mahabang panahon ay unti-unting binuldoser ng mga komponent ng Kaliwa ang pundasyon ng diktadura hanggang sa tuluyan na itong magiba ng People Power (Branigin, 1981; Nemenzo, 1985; Bagadion, 1986; Ocampo, 2011; Sison, 2012; Ellao, 2015; Araullo, 2016; Palatino, 2016), kaya't hindi dapat isantabi ang papel ng armadong pakikibaka (na konektado at humuhugot ng lakas mula sa tunggalian ng mga uri) laban sa diktadura.

Samantala, may pahiwatig ang "Sayaw sa Bubog" ng The Jerks hinggil sa pag-iral ng tunggalian ng mga uri sa panahon ng EDSA I na itinuturing nitong "palabas na moro-moro," isang kaduda-dudang pangyayari sa kanilang paningin ("ito kaya ay totoo?") kaya naman itinatanong nila kung para "kanino" ang sinasabing "pagbabago" sa EDSA (Pura, 1997). Nasa awit mismo-sa unang saknong — ang sagot sa kanilang sariling tanong. "Bumaha ng pangako" sa panahon ng EDSA ngunit "tuloy sa pagkakapako" ang mga mamamayang Filipino, "may utang pati apó" (Pura, 1997) dahil sa pagtutol ng pumalit sa diktador sa panawagang debt repudiation sa mga inutang ng diktadurang Marcos. Sa isyu ng reporma sa lupa (pamamahagi ng lupa sa mga tagabungkal o land to the tiller), nilinaw ng "Sayaw sa Bubog" (Pura, 1997) na "tuloy ang ligaya sa iba't ibang hacienda" dahil ang mga panginoong maylupa o mga landlord (gaya ng pangulong pumalit sa diktador) ay panginoon pa rin. Hindi pa ganap na naipatutupad ang pamamahagi ng lupa sa mga nagbubungkal nito kaya naman ang mga "manggagawa't, magsasaka" ay "kumakalam" pa rin "ang sikmura." Dapat bigyan ng diin na ang "reporma sa lupa" ng diktadurang Marcos at ng unang administrasyong Aquino ay kapuwa hindi naging matagumpay (Wurfel, 1977; Kerkvliet, 1974; " Hacienda Dolores: Testament to CARP failure," 2014) gaya ng pinatutunayan ng pagkakaroon ng Comprehensive Agrarian Reform Program Extension with Reforms (CARPER) sa ilalim ng administrasyong Macapagal-Arroyo at ikalawang administrasyong Aquino (Ellao, 2014; Tadem, 2015; " CARP, CARPER: Failing, falling, dead?." 2015). Katunayan, itinutulak din ng ilang aktibistang grupo ang pagsasabatas ng mas radikal na Genuine Agrarian Reform Bill o GARB (" KMP tells Congress to bury the long-dead CARP, legislate GARB," 2018). Kung nagtagumpay ang programa sa reporma sa lupa ng diktadurang Marcos at ng unang administrasyong Aquino, hindi na kakailanganin pa ang CARPER o kaya'y ang GARB.

Naniniwala ang The Jerks, gaya ni Anderson, na administrasyong cacique rin ang unang rehimeng Aquino tulad ng diktadurang Marcos kaya walang nagbago sa kalagayan ng mga simpleng mamamayan pagkatapos ng EDSA I. Ganito rin ang pinatutunayan ng komprehensibong pagtalakay ni Arillo (2000) sa kalagayan ng bansa sa ilalim ng dalawang rehimeng post-EDSA I (unang administrasyong Aquino at administrasyong Ramos) sa pamamagitan 
ng aklat na Greed and Betrayal. Ang tahas na pagbibigay ng diin ng The Jerks sa mga isyung sosyo-ekonomiko (utang, reporma sa lupa, "nakawan" at "karahasan") na hindi naresolba ng EDSA I ay isang pagtatangkang ibalik "sa ibabaw ng mesa" - sa adyenda ng pamahalaang nakaupo sa kabesera at sa adyenda mismo ng mga mamamayang tila "naglalakad nang tulog" kaya naman "tiyak na mauumpog," madudurog sa pagbabanggaan ng iba't ibang puwersang politikal-ang mga usaping nakaaapekto sa buhay ng mga ordinaryong mamamayan na siyang nagbigay-kapangyarihan sa bisa at tagumpay ng EDSA I. Pinatingkad ng The Jerks ang "pangangalampag" o "panggigising" sa kamalayan ng mga mamamayan at ng pamahalaan sa pamamagitan ng paggamit ng awit sa ikatlong panauhan o third person na akmang-akma kapag ang layuni'y ilarawan ang kasalukuyang kalagayan ng lipunan (gaya ng karaniwang pananaliksik na gumagamit ng paraang deskriptibo na umiiwas sa paggamit ng "ako" upang higit na maging tunogawtoritatibo).

Hindi rin lantad ang pagbanggit ng "Kumusta $\mathrm{Na}$ " sa tunggalian ng mga uri. Ang perspektiba nito ay isinulat sa porma ng pakikipagdiyalogo ng sumulat sa isang kalahok sa EDSA I (ang taong may hila-hilang kariton). Ang kariton ay simbolo ng pagdaralita sa Pilipinas (ito ang stereotypical na gamit ng mga magbabakal-bote na kakarampot lang ang kita sa pagbili at pagbibili/pagbebenta ng mga junk/scrap). Sa mga nakaraang taon, ang kariton ay simbolo na rin ng kawalan ng tirahan o homelessness: ang mga walang matirhan ay sa kariton tumitira, dala-dala ang kakaunti nilang ari-arian. Malinaw kung gayon, na sa pananaw ng sumulat ng "Kumusta $\mathrm{Na}$," ang EDSA I ay isang pag-aalsang nilahukan ng maraming mahihirap, kaya nga isang mahirap, isang taong may hila-hilang kariton ang piniling "kumustahin" ng Yano, ilang taon pagkatapos ng "mapayapang rebolusyon." Dekada 90 na nang isulat ang "Kumusta $\mathrm{Na}$ " kaya sa panahong iyon, may dahilan ang Yano na itanong sa taong may kariton kung "kumusta na" siya at kung "ayos pa ba ang buhay natin," kung "kaya pa ba" ng may kariton na magpatuloy sa paglalakbay sa buhay na tila wala namang ipinagbago ilang taon pagkatapos ng "rebolusyon."

Lalong tumingkad ang pag-iral ng tunggalian ng mga uri sa obserbasyon ng Yano na "nakita kita (ang taong kausap na dati'y lumahok sa EDSA) kahapon/may hila-hilangkariton/huminto sa may Robinson/tumanga buong maghapon/sikat ka noon sa TV/kasi, kasama ka doon sa rali/pero ngayo'y nag-iisa/naglalakad sa may Edsa" (Abay, 1994). Naglalakad pa rin sa EDSA ang taong may kariton-ang simbolo ng mga ordinaryong mamamayan na naniwala sa pangako ng EDSA-ngunit ilang taon pagkatapos ng "rebolusyon," mag-isa na lamang siya at tila iniwan (o napag-iwanan) na ng mga "mayaman" na kasama noon sa "rebolusyon" ayon sa "Handog ng 
Pilipino sa Mundo." Di man tahas, ang "Kumusta Na" ay nagpapahiwatig ng panunumbalik ng "mayaman," ng elite, ng mga cacique sa kanilang lumang gawain bago ang EDSA I: ang panatilihin ang status quo na hindi lahat ay may oportunidad na umunlad ang buhay. Para sa Yano, ang tunggalian ng mga uri ay malinaw na makikita pa rin sa kalsada, sa kahubaran ng mga bagaybagay, sa lugar na nilalakaran ng taong may hila-hilang kariton, sa labas ng Robinson at iba pang ilusyon ng kaunlaran. Ang halos di nagbabagong malawak na agwat o gap sa pagitan ng mga mahihirap na walang-wala at ng mga mayayaman na nagtatamasa ng maganda at maluhong buhay sa kabila ng pagpapalit-palit ng mga kontemporanyong administrasyon ay pinatutunayan din ng estadistika hinggil sa income share ng upper $50 \%$ at bottom 50\% ng populasyon batay sa datos sa presentasyon ni Africa (2011), former administrator ng National Statistics Office (NSO), na pinamagatang "Family Income Distribution in the Philippines, 1985-2009: Essentially the Same":

\section{Pananaw sa EDSA I:}

\section{Euphoria sa Pansamantalang Pagkakaisa, Tuloy-Tuloy na Pakikibaka at Landas ng Bahala $\mathrm{Na}$}

Lunod na lunod sa euphoria ang mga titik ng "Handog ng Pilipino sa Mundo" dahil isinulat ito pagkatapos na pagkatapos ng EDSA I noong 1986. Sa

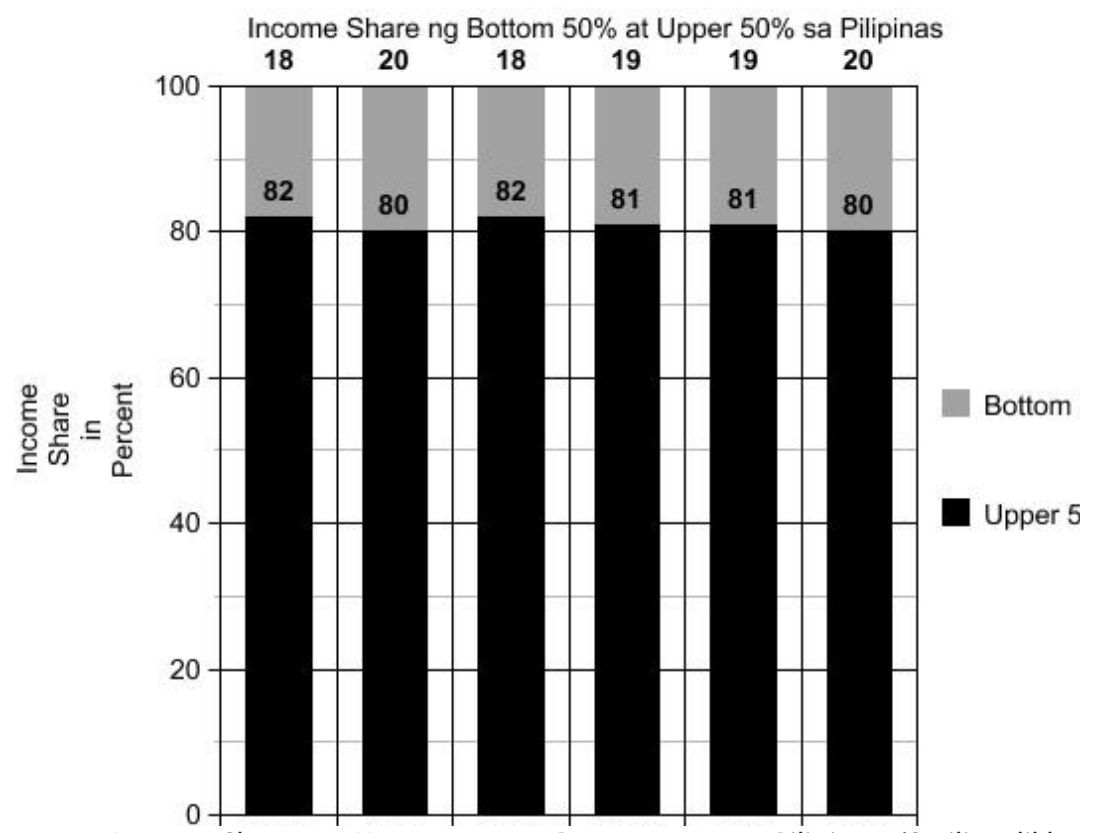

Larawan 1: Income Share ng Upper $50 \%$ at Bottom $50 \%$ sa Pilipinas. (Sariling likha ng mananaliksik ang talahanayan batay sa datos sa presentasyon ni Africa, 2011). 
panahong iyon, namamayani ang galak ng buong sambayanang Filipino na buong-pagmamalaking itinatanghal sa mundo ang kaniyang "handog," ang diumano'y "mapayapang paraang pagbabago" (Paredes, 1986). "Kay sarap palang maging Pilipino" ayon sa awitin dahil sa pamamagitan ng pagkakaisa ng mga mamamayan laban sa diktadura, "naging langit itong bahagi ng mundo." Nangangaral ito sa bawat isa na maging mapagmatyag at "Huwag muling payagang umiral ang dilim" na namayani sa panahon ng diktadura. Kapuri-puri ang paggamit ng "dilim" para tukuyin ang diktadura sapagkat binibigyan ng diin nito ang matitinding paglabag sa karapatang pantao na naganap sa panahon ng diktadurang Marcos, taliwas sa propaganda ng mga rebisyonista na diumano'y mahusay na pinuno si Ferdinand E. Marcos (pansinin halimbawa ang propagandang video na inupload ni Asingan Pangasinan sa YouTube noong 2012). Nakatala sa Wall of Remembrance sa Bantayog ng Mga Bayani sa Quezon City (malapit sa MRT Quezon Avenue Station) ang mga bayaning nagbuwis ng buhay o nag-alay ng kanilang pawis at pagod upang ibagsak ang mapaniil na diktadurang Marcos. Masisipat sa link na ito ang ilan sa mga pangalan ng mga bayaning ito: http://bantayog. wordpress.com/the-wall/. Marami ring aklat, na gaya ng Martial Law in the Philippine: My Story ni Senador Aquilino "Nene" Q. Pimentel, Jr. (2006); Conjugal Dictatorship of Ferdinand and Imelda Marcos ni Primitivo Mojares (1986); at Some are Smarter Than Others: The History Of Marcos' Crony Capitalism ni Ricardo Manapat (1991), ang nagpapatunay sa pag-iral ng "dilim" sa panahon ng Batas Militar.

Upang hindi na muling "umiral ang dilim," itinatanghal ng "Handog ng Pilipino sa Mundo" (Paredes, 1986) ang liwanag ng demokrasyang pampulitika: “Tinig ng bawat tao'y bigyan ng pansin." Para sa Apo, sapat nang magkaroon ng partisipasyon sa prosesong politikal ang masa upang mahadlangan ang panunumbalik ng diktadura. Walang pahiwatig ang awit kung paano aktuwal na makakamtan ang "katarungan" na kaakibat ng "katotohanan" at "kalayaan" sa pamamagitan lamang ng pagiging "magkasama" ng "mahirap" at "mayaman" bilang mga "magkakapatid" "sa Panginoon." Sa pananaw ng nagsulat ng "Handog ng Pilipino sa Mundo" sapat nang dahilan para ganap na magdiwang ang panunumbalik ng demokrasyang pampolitika. Nagtapos ang awit sa pag-asang posible ang lahat "Basta't magkaisa tayong lahat."

Samantala, malinaw na malinaw ang kritisismo ng "Sayaw sa Bubog" sa kabiguan ng EDSA I na magdulot ng aktuwal na repormang sosyoekonomiko sa buhay ng mga ordinaryong Filipino sapagkat naisulat ang awit na ito noong dekada 90 na. Para sa The Jerks, isang "kasinungalingan" at "kahangalan" ang pangakong "kalayaan" ng EDSA I (Pura, 1997) dahil hindi ganap ang kalayaang ito. "Bumaha" lamang "ng pangako," at ang 
"lason" ng pangingibabaw ng mga dayuhan at ilang elite o cacique sa ekonomiya at politika ng bansa ay patuloy na "isinubo" sa mga mamamayan kaya naman "tuloy sa pagkakapako" sa "utang pati apo" ng mga lumahok sa EDSA. Katunayan, tinanggap ng unang administrasyong Aquino ang responsabilidad sa pagbabayad ng mga inutang ng diktadurang Marcos kahit pa nga malinaw na ang maraming proyektong inutang nito gaya ng isang bilyong dolyar na Bataan Nuclear Power Plant ay maanomalya (African Forum and Network on Debt and Development, 2007). Para sa The Jerks, ang EDSA I ay isa lamang "palabas na moro-moro" ng mga naghaharing-uri, isang pagpapalit lamang ng nasa puwesto ngunit hindi ng mga programang sosyo-ekonomiko gaya ng pribatisasyon, deregulasyon, at liberalisasyon na patuloy na nagpapahirap sa mga mamamayan. Ayon sa awit (Pura, 1997), ang mga mamamayan ay tila nagsasayaw "sa bubog" at kung hindi magigising sa katotohanan na walang pagbabagong idinulot ang EDSA I, kung magpapatuloy sa paglalakad "nang tulog ay tiyak na mauumpog." Bakas ang galit ng awit sa kawalan ng demokrasyang pangekonomiya sa panahong "tuloy ang ligaya sa iba't ibang hacienda" habang ang mga "manggagawa't, magsasaka, kumakalam" pa rin "ang sikmura." Makabuluhan din ang pagbanggit ng awit sa pagpapatuloy ng "kaguluhan, nakawan, karahasan" sa panahon ng sinasabing "bagong demokrasya" pagkatapos na mapatalsik ang diktadura. Para sa The Jerks, wala pa ring kaayusan sa lipunan, mayroon pa ring katiwalian dahil hindi nakikinabang ang lahat ng mamamayan sa umiiral na sistema at ang mga nakikibaka para sa pagbabago ay karaniwang pinapatawan pa rin ng "karahasan" ng gobyerno. Gaya ng ipinakikita sa obra maestra ni Lino Brocka na Ora Pro Nobis (1989), nagpatuloy sa panahon ng unang administrasyong Aquino ang pamamaslang sa mga aktibista na pinagbibintangang kasapi ng kilusang komunista sa bansa. Batay sa tala ni Arillo (2000), nagpatuloy ang malalang paglabag sa karapatang pantao sa ilalim ng unang administrasyong Aquino. Ang "dilim" na inasahan ng "Handog ng Pilipino sa Mundo" na hindi na muling iiral ay muling nanumbalik. Halimbawa, dokumentado ang marahas na dispersal sa mga magsasakang nagrali sa Mendiola noong 1987 upang igiit ang pagkakaroon ng reporma sa lupa (Richburg, 1987; Espada, 2004; Curaming, 2013). Sa tala ng Task Force Detainees of the Philippines (TDFP), kagimbal-gimbal ang estadistika ng mga paglabag sa karapatang pantao sa panahon ng unang administrasyong Aquino: "more than 1.2 million victims of dislocations due to military operations, 135 cases of massacres, 1,064 victims of summary executions, and 20,523 victims of illegal arrest and detention" (Olea, 2009, para. 5). Kaugnay ng mga masaker ng mga magsasakang nakikipaglaban para sa kanilang karapatan at/o reporma sa lupa, ilan sa mga rehimeng post-EDSA ay may pantapat sa mga masaker 
ng rehimeng Marcos gaya ng Escalante massacre (Doyo, 2016; "Escalante Massacre," 2015): Mendiola massacre sa ilalim ng unang administrasyong Aquino (Lapeña, 2012), at Hacienda Luisita massacre (Tuazon, 2004) sa ilalim ng rehimeng Macapagal-Arroyo at Kidapawan Massacre sa ilalim ng ikalawang administrasyong Aquino.

Nagtapos ang "Sayaw sa Bubog" sa pagbibigay-diin ng chorus na nagpapaalalang hindi dapat maglakad "nang tulog" ang mga mamamayan upang hindi na patuloy na "maumpog." Ito'y isang panawagan na ituloy ang pakikibakang sinimulan sa EDSA I (at mas maaga pa rito) upang makamtan ang isang lipunang lahat ay nagtatamasa ng demokrasya di lamang sa larangan ng politika, kundi pati sa larangan ng ekonomiya, isang bansa na ang bawat isa'y nagtatamasa ng karapatan sa desenteng pamumuhay na karaniwang tinatamasa lamang ng mga mayayaman sa lipunan. Hindi kataka-taka na hanggang sa mga kilos-protesta sa panahon ng rehimeng MacapagalArroyo at sa panahon ng ikalawang administrasyong Aquino, patuloy na inaawit ng The Jerks ang "Sayaw sa Bubog" na paboritong-paborito rin ng mga raliyista dahil sa makabuluhang mensaheng nananatiling makabuluhan sapagkat halos walang pagbabagong makabuluhan sa pagpapalit ng mga administrasyon mula noon hanggang ngayon. Saksi ang mananaliksik sa di mabilang na kilos-protesta na nilahukan ng mga mamamayang naghahangad pa rin ng ganap na katuparan ng mga pangako ng EDSA I: sa tuwing aawit ang The Jerks, hindi maaaring hindi awitin ang "Sayaw sa Bubog" dahil tuloy ang pagsasayaw ng masa sa bubog, tuloy ang pagsasakripisyo nila sa pamamagitan ng walang katapusang pakikibaka upang makamit ang mga pangako ng EDSA I na tinalikuran na maging ng mga pangunahing nakinabang dito.

Negatibo rin ang pananaw ng "Kumusta $\mathrm{Na}$ " sa pangmatagalang epekto ng EDSA I. Pagkatapos "kumustahin" ang buhay ng mga ordinaryong mamamayan ilang taon pagkatapos nilang lumahok sa EDSA, ginunita ng Yano ang masasayang tagpo sa "rebolusyon" (Abay, 1994): "Napanood kita sa TV/Sumama ka sa rali/Kasama mga madre/Pinigilan mga tangke/ Umiiyak ka pa/Sa harap ng mga sundalo/Namigay ka pa ng rosas/Na nabili mo sa kanto." Ipinaalala rin ng awit ang papel ng pananampalataya sa EDSA I: "Dala-dala mo pa/Estatwa ni Santo Niño/Eskapularyo't Bibliya/At sangkatutak na rosaryo/At sa gitna ng EDSA/Lumuhod ka't nagdasal pa/ Our Father, Hail Mary,/from the bounty/through Christ our Lord, Amen." Sa pamamagitan ng isang pagpapatawa ay binigyan ng diin din ng awit ang popularidad ng EDSA I: "Pebrero bente sais/Nang si Apo ay umalis/Ngiti mo'y hanggang tenga/Sa katatalon/Napunit ang pantalon mo/Pero hindi bale/Sabi mo/Marami naman kame..." Katunayan, hindi lamang sa EDSA nagtipon-tipon ang mga tao upang pabagsakin ang diktadurang Marcos. 
Isang kahunghangan ang propaganda ng mga rebisyonistang maka-Marcos na nagsasabing sa "Imperial Manila" lamang mayroong protesta("Imperial Manila: Uniting the Nation at a Price," 2016; Doronila, 2006). Sa lahat ng mga sentrong bayan at lungsod sa Luzon, Visayas, at Mindanao ay may mga kilos-protesta laban sa diktadura kasabay ng nagaganap sa EDSA (Luz, 2016) kaya nga hindi na alintana ng mga nagdisko sa kalye kung mapunit man ang pantalon nila dahil "marami naman" sila. Gayunman, di gaya ng "Handog ng Pilipino sa Mundo," hindi nagkasiya ang "Kumusta Na" sa paggunita sa tamis ng tagumpay ng EDSA. Sa mga huling bahagi, binigyan ng diin ng awit ang "pag-iisa" ng ordinaryong mamamayang lumahok sa EDSA_ilang taon pagkalipas ng "rebolusyon" - na tila pinabayaan na ng pamahalaan kaya ngayo'y "May hila-hilang kariton/Huminto sa may Robinson/Tumanga buong maghapon" dahil walang bahay at wala ring trabaho. Ngunit, "naglalakad" pa rin sa EDSA ang taong may hila-hilang kariton. Gayunman, di gaya ng paninindigan sa awit ng The Jerks, hindi gaanong malinaw ang landas na dapat tahakin ng mamamayan ayon sa Yano. Sa tatlong ulit ng "bahala na" nagtapos ang "Kumusta Na": isang pagtitiyak o assurance na may magbago man o wala, may pakikibaka man o wala, tuloy pa rin ang buhay ng mga ordinaryong mamamayan upang likhain ang kanilang kasaysayan labas sa ilusyon ng kaunlaran na inilalako ng mga kontemporanyong cacique sa lipunan-isang pakikibakang tunay ring pakikibaka, ang mabuhay at umiral sa gitna ng sitwasyong walang kabuhayan at tila walang pagbabago ang sitwasyon ng kaniyang pag-iral.

Sa pangkalahatan, nalagpasan ng awit ng The Jerks at ng Yano ang euphoria ng EDSA Uno, dahil na rin sa panahon na naisulat ang mga ito (halos isang dekada pagkatapos ng EDSA Uno) ay lantad na lantad na ang pagpapatuloy ng mga suliraning iniwan ng rehimeng Marcos at nabigong lutasin (kundi man sinusubukan pa ring lutasin sa pamamagitan ng parehong paraang ginamit ng diktadura) ng mga administrasyong postMarcos. Halimbawa, kaugnay ng perenyal na suliranin ng kawalan ng trabaho, ang pag-e-eksport ng mga manggagawang Filipino na malawakang ipinormalisa ng rehimeng Marcos ay patuloy na isinulong ng mga rehimeng post-EDSA sa kabila ng mabibigat na social cost nito (San Juan, 2014). Gayundin, hanggang sa kasalukuyan, wala pang presidenteng postMarcos na nagtangkang ibasura ang Presidential Decree No. 1177 (utos sa awtomatikong paglalaan ng badyet para sa pagbabayad-utang) ni Marcos ("Revising the budget process in order to institutionalize the budgetary innovations of the new society," 1977). Patuloy sa pagkakabaon sa utang ang bansa gaya ng pinatutunayan ng balitang malapit nang umabot sa 8 trilyong piso ang utang ng Pilipinas sa ilalim ng administrasyong Duterte (De Vera, 2018). Higit sa lahat, ang monopolyo ng iilang angkan sa sistemang politikal 
at ekonomiko ng bansa-na kitang-kita na sa panahon ng pamamasasa ng diktadurang Marcos at ng mga kroni nito sa yaman at kapangyarihan-ay malinaw na hindi pa rin natitibag, sukdulang halos mawalan na ng gana ang marami-raming ordinaryong mamamayan sa pagsariwa sa diwa ng EDSA Uno sa mga nakaraang taon (Mydans, 2006; Ylagan, 2018; "Parsing Edsa,"2019). Sa pagtangan ng mga rehimeng post-EDSA sa parehong mga eskemang ekonomiko ng diktadurang Marcos-neoliberal na kapitalismong nagpapalala sa dependensiya ng Pilipinas sa mga dayuhan at patuloy na nagpapayaman sa iilang angkan (San Juan, 2016; Ocampo, 2017)_ang bansa ng 18 bilyonaryo sa dolyar ("Philippines' 50 Richest," n.d.) ay may humigit-kumulang 22 milyong anak-dalitang mamamayan sa kasalukuyan sa rekord mismo ng gobyerno ("Philippines' Poverty Rate Declines; More Well-Paying Jobs and Opportunities Needed," 2018) na nakabatay sa mababang pamantayan sa pagtatakda ng antas ng kahirapan. Noong Enero 2018, ang antas ng kawalan ng trabaho at underemployment ng Pilipinas ayon sa Philippine Statistics Authority (PSA) ay 5.3\% at 18.0\% ("Employment Situation in January 2018 (Final Results)," 2018). Sa datos naman ng World Bank, malinaw na makikita ang halos hindi nagbabagong estadistika sa porsiyento ng pambansang kita na hawak ng pinakamayamang 40\% ng populasyon sa ilalim ng mga rehimeng post-EDSA (1991-2015) ("Distribution of Income or consumption by quintile," 2015):

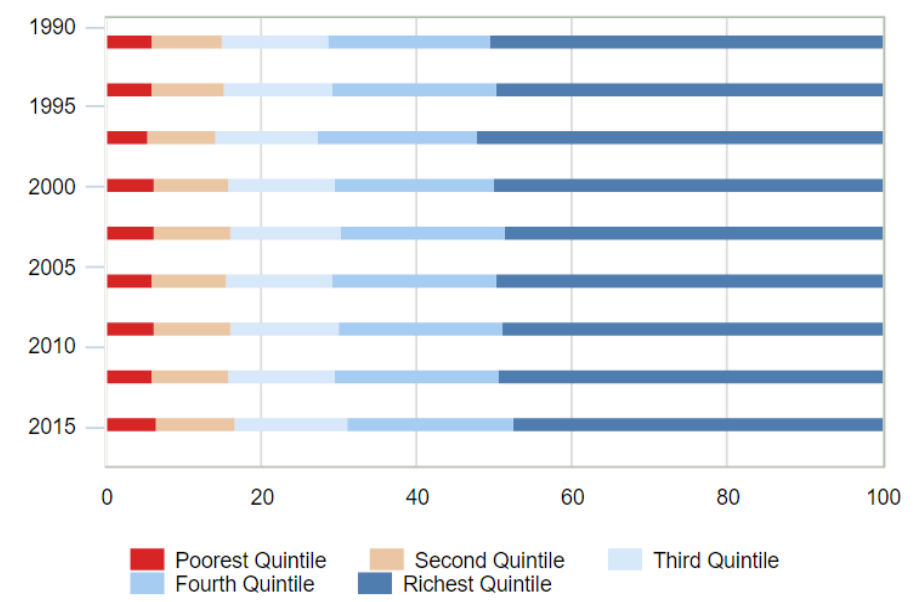

Larawan 2. Distribusyon ng kita ng iba't ibang uring panlipunan sa Pilipinas ("Poverty and Equity Data Portal," n.d.).. 


\section{Kongklusyon}

Sinasalamin ng mga makasaysayang awit ng bansa ang nakaraan, kasalukuyan, at hinaharap ng sambayanan gaya ng ipinakita sa pagsusuri ng tatlong awiting isinulat pagkatapos ng EDSA I. Dakilang monumentong nagpapaalala sa dilim ng diktadurang Marcos ang "Handog ng Pilipino sa Mundo," "Sayaw sa Bubog" at "Kumusta $\mathrm{Na"} \mathrm{sapagkat} \mathrm{binibigyan} \mathrm{ng} \mathrm{diin}$ ng tatlong awiting ito na minsan sa ating kasaysayan, nagsama-sama ang maraming mamamayan upang pangibabawan ang kanilang takot at tuluyang ibagsak ang diktador sa pamamagitan ng kolektibong pagkakaisa at pagkilos. Kung nagpokus ang "Handog ng Pilipino sa Mundo" sa pagdiriwang ng panunumbalik ng demokrasyang pampolitika, pinapaalalahanan naman tayo ng "Sayaw sa Bubog" at "Kumusta Na" na dapat ipagpatuloy ang pakikibaka o ang paghahanap ng daan tungo sa inaasam na tunay na pagbabago di lamang sa sistemang pampolitika, kundi maging sa sistemang pangekonomya ng bansa. Mananatiling "di tapos ang rebolusyon," mananatiling walang katuparan ang pangako ng EDSA I, II at mga susunod pang EDSA hangga't hindi nagiging ganap ang kalayaan ng mga mamamayan sa aspektong politikal at ekonomiko-hangga't may mga mamamayang may hila-hilang kariton na tumatanga lamang sa buong maghapon sa labas ng mga Robinson at iba pang ilusyon ng kaunlaran, at hangga't ang mga may-ari lamang ng asyenda ang maligaya. Sabi nga sa isa pang awiting pinamagatang "Tatsulok" (orihinal ng bandang Buklod na ni-revive ng Bamboo), "habang may tatsulok" (social pyramid), "hindi matatapos itong gulo."

Bagama't may espasyo na sa midya at paaralang mainstream ang mga ganitong awitin, tungkulin ng bawat mulat na mamamayan na linawin ang konteksto ng mga awiting ito-kasama na ang ideya ng paglagpas sa pagsariwa lamang sa panunumbalik ng politikal na demokrasya tungo sa pagbabandila ng mga radikal na repormang sosyo-ekonomiko upang hindi na maging bansa ng malalawak na agwat ng mga mall at kariton (gaya ng pahiwatig sa awit na "Kumusta Na") at ng mga magsasaka at asendero (gaya ng banggit sa "Sayaw sa Bubog") ang Pilipinas. Imulat din ang iba pang nabubulagang mamamayan hinggil sa tagumpay at nararapat na pagpapalawak pa ng saklaw ng EDSA I at ng iba pang pakikibaka ng sambayanang Filipino. Ang pangangailangang isabalikat ang gayong gawain ay binigyang-diin mismo ni Paredes (2016) sa pagsariwa sa ambag ng kaniyang awitin na makabuluhan pa rin sa kasalukuyan: "May it inspire the present generation of Filipinos who are too young to have experienced EDSA, and too uninformed about its significance to the freedoms they enjoy. People think that the power to move a nation lies in the hands of politicians" (para 19).

Ang inaakala ng mga nakatatandang henerasyon na madaling maunawaang katotohanan mula sa mga awiting tinalakay at sa kasaysayang 
kaugnay nito ay unti-unti nang nawawaglit sa kamalayan ng mga henerasyong post-EDSA. Batay mismo sa karanasan ng mananaliksik sa pagtuturo sa mga pribadong paaralang gaya ng Colegio San Agustin-Makati at De La Salle University, Manila, marami-raming salita sa lyrics ng mga awiting ito ay mahirap nang unawain para sa mga estudyante (dahil na rin sa kakulangan nila ng sapat na kultural na eksposyur sa wikang pambansa) at halos wala nang estudyante ang nakakakilala sa mga pigurang politikal gaya nina Senador Lorenzo Tañada at Jose W. Diokno na pawang nasa music video ng "Handog ng Pilipino sa Mundo" bilang mga beterano ng kilusang anti-Marcos at ng mga kilusang panlipunan na nakikibaka rin para sa mas malawakang pagbabagong panlipunan sa ekonomiya, kaya kapuwa iginagalang at tinitingala maging ng mga puwersa sa Kaliwa sa bansa. Bukod dito, noong 2016, nanguna o pumangalawa ang anak ng diktador sa mga mock poll para sa kandidato sa eleksiyong bise-presidensiyal sa mga unibersidad gaya ng University of Santo Tomas, Arellano University, Ateneo de Manila University at De La Salle University (De Vera, 2016). Hindi rin dapat kalimutan na 14,155,344 boto ang nakamit ni Bongbong Marcos noong 2016, kompara sa 14,418,817 boto para kay Leni Robredo ("Final results: 2016 presidential and vice presidential canvass," 2016). Kung wala mang historical amnesia ay masasabi namang tila nag-move on na ang maraming Filipino kaya nagagawa na nilang iboto maging ang anak ng diktador na noo'y nakadestiyero kasama ng kaniyang angkan. Habang lumilipas ang mga dekada, namemeligrong mawala sa kamalayan ng nakararami ang mga aral ng kasaysayan at maaaring manumbalik muli ang dilim ng diktadura. Sa ganitong diwa, mainam na gamot sa historical amnesia at historical revisionism ng mga nabubulagang maka-Marcos (at maka-Aquino na rin) ang tatlong awiting sinipat sa papel na ito.

Sa minimum, dapat ipreserba ng mga mulat na mamamayan ang gunita, ang alaala, ang mismong pag-awit sa mga awiting ito sapagkat, gaya ng sinabi ni Milan Kundera (1996): Ang pakikibaka ng sangkatauhan laban sa diktadura ay pakikibaka ng gunita laban sa pagkalimot. ["The struggle of mankind against tyranny is the struggle of memory against forgetting."] (p. 4). Bukod sa mga awiting tinalakay sa artikulong ito, maaari ring ilahad ang detalyadong konteksto ng "Saranggola ni Pepe" na sinulat ni Nonoy Gallardo at inawit ni Celeste Legaspi (awiting may mga pahiwatig ng patama sa diktadurang Marcos, ayon mismo kay Legaspi batay sa video ng TV5 InterAksyon na ini-upload sa YouTube noong 2016) at ng "Pagbabago" (tungkol sa People Power at sa kabiguan nito na baguhin ang búhay ng nakararami dahil sa pangingibabaw ng elite) ni Jess Santiago (2008). Marami pang katulad na awitin ang nalilikha sa bawat panahon ng ating kasaysayan.

Sa panahon ng administrasyong Duterte na tila ganap nang 
nakapanumbalik sa poder ang mga Marcos (rurok ang matagumpay na paglalagak ng mga labi ni Marcos sa Libingan ng mga Bayani sa kabila ng malawakang protesta ng sambayanan, at ang pagiging pangalawa ni Marcos, Jr. sa eleksiyong bise-presidensiyal at pagkahalal na senador ng isa pang anak ni Marcos noong 2019), lalong nagiging imperatibo ang gayong adhikain. Magtagumpay man ang mga rebisyonista at may amnesya na "rebisahin" ang kasaysayan upang itampok bilang "bayani" o "magaling na pinuno" ang diktador na si Ferdinand Edralin Marcos o kaya'y sabihin na santo at perpekto ang unang administrasyong Aquino, laging naririyan ang mga makasaysayang awiting post-EDSA I upang ipaalala sa madla ang mga tunay na naganap at ano pa ang dapat maganap sa kasaysayang nililikha ng bayan mismo. 


\section{MGA SANGGUNIAN}

Abay, D. (1994). Kumusta Na. [Recorded by Yano] On Yano [CD]. San Juan City: Alpha Records.

Abinales, P. N., \& Amoroso, D. J. (2005). State and society in the Philippines. Pasig City: Anvil Publishing, Inc. Adams, W. (2003). Where is the Love? [Recorded by Black Eyed Peas]. On Elephunk [CD]. California: A \& M.

Adams, N. (2013, April 28). The inspiring force of "We shall overcome." Retrieved from https://www.npr. org/2013/08/28/216482943/the-inspiring-force-of-we-shall-overcome

Africa, T. (2011, March 18). Family income distribution in the Philippines, 1985-2009: Essentially the same. Retrieved from https://www.sws.org.ph/downloads/publications/pr20110321\%20-\%20Family\%20 Income\%20Distribution\%20by\%20Mr\%20Tomas\%20Africa_FINAL.pdf

African Forum and Network on Debt and Development (AFRODAD). (2007). Illegitimate debt \& underdevelopment in the Philippines: A Case Study. Retrieved from http://s3.amazonaws.com/ zanran_storage/www.afrodad.org/ContentPages/43088398.pdf

Amoneth. (2010, May 22). Bella ciao by Jose Maria Sison [Video file]. Retrieved from https://www.youtube. com/watch?v=PsDNAPgZJiA

Anderson, B. (1988, May-June). Cacique democracy and the Philippines: Origins and dreams. New Left Review 169, 3-33. Retrieved from https://newleftreview.org///169/benedict-anderson-caciquedemocracy-and-the-philippines-origins-and-dreams

Andrada, M. (2016, March 8). Pagdugtong sa awit na "Babae" ng Inang Laya. Pinoy Weekly. Retrieved from http://pinoyweekly.org/new/2016/03/babae-inang-laya/

Aquino, B. (1987). Politics of plunder: The Philippines under Marcos.Quezon City: Great Books Trading and the University of the Philippines College of Public Administration.

Arillo, C. (2000). Greed \& betrayal: The sequel to the 1986 EDSA revolution. Las Piñas: CTA \& Associates.

Araullo, C. (2016, February 28). Legacy of EDSA 'People Power'. Retrieved from https://www.bulatlat. com/2016/02/28/legacy-of-edsa-people-power/

Artists for the removal of Gloria (ARREST Gloria). (2006, August 5). The challenges of our times. Retrieved from https://www.bulatlat.com/2006/08/05/the-challenges-of-our-times/

Asingan Pangasinan. (2012, September 21). Untold story of Martial Law (Aquino - Marcos). Retrieved from https://www.youtube.com/watch?v=P6lmb00sqyY.

Ashton, M. (2011, March 10). Great political poems (No.5):The Silesian weavers [blog post]. Retrieved from http://drmatthewashton.com/2011/03/10/great-political-poems-no5-the-silesian-weavers/

Ayroso, D. (2015, October 30).\#Manilakbayan2015 turns UP grounds into cultural, political hub. Retrieved from https://www.bulatlat.com/2015/10/30/manilakbayan2015-turns-up-grounds-into-culturalpolitical-hub/

Bagadion, B. (1986). People power in the Philippines. Retrieved from http://lynchlibrary.pssc. org.ph:8081/bitstream/handle/0/926/05_People\%20Power\%20in\%20the\%20Philippines. pdf? sequence $=1$ \&isAllowed $=y$

Bantayan, O. (2017, February 26). What's eating Jim Paredes?. Retrieved from http://davaotoday.com/ main/stand-point/standpoint-whats-eating-jim-paredes/

Boyce, J. K. (1993). The Philippines: The political economy of growth and impoverishment in the Marcos era. Hawaii: University of Hawaii Press. 
Branigin, W. (1981, November 22). Philippine far left grows as center defers to Marcos. Retrieved from https://www.washingtonpost.com/archive/politics/1981/11/22/philippine-far-left-grows-ascenter-defers-to-marcos/4aec5675-11f7-4663-a160-15a76942b715/?noredirect=on\&utm_ term $=.63 \mathrm{fd} 9 \mathrm{dd} 8 \mathrm{a} 1 \mathrm{de}$

Brazill, L. (2009, September 4). Bread \& roses:The song [blog post]. Retrieved from http://eachlittleworld. typepad.com/each_little_world/2009/09/bread-roses-the-song.html.

Buhle, M.J. \& P. Buhle. (2012, April 9). The historic Wisconsin solidarity sing along. Retrieved from https:// progressive.org/dispatches/historic-wisconsin-solidarity-sing-along/

Cabling, P. (2013). A new and better way: The people's anthem [video file]. Retrieved from https://www. youtube.com/watch?v=pZtKpfHNrL8\&t=11s.

Caparas, G. (2004, May 9). Songs in the key of politics. Retrieved from http://pcij.org/stories/songs-in-thekey-of-politics/

CARP, CARPER: Failing, falling, dead?. (2015, June 11). Retrieved from https://pcij.org/blog/2015/06/11/ carp-carper-failing-falling-dead

Caruncho, E. (2018, July 22). Dong Abay's state of the nation address. Retrieved from https://www.inquirer. net/sona2014/article/300843?c=8

Castro, C. (2011). Musical renderings of the Philippine nation. New York: Oxford University Press.

Ching, M. A. (2011, February 25). Stars at the EDSA people power of 1986. Retrieved from http://www.pep. $\mathrm{ph} /$ news/28455/stars-in-the-edsa-people-power-of-1986

Curaming, R. (2013). The end of an illusion: Mendiola massacre and political pransition in post-Marcos Philippines. In N. Ganesan \& S, C. Kim (Eds.), State violence in East Asia. Lexington: University of Kentucky Press.

Curaming, R. \& L. Claudio. (2010). A historicised (re)assessment of EDSA 'People power' (1986). ARI working paper series no. 134. Retrieved from www.ari.nus.edu.sg/pub/wps.htm

De Lara, A. (2008, August 2). Street repertory: The Role of Art in the People's SONA. https://www.bulatlat. com/2008/08/02/street-repertory-the-role-of-art-in-the-people\%E2\%80\%99s-sona/

De Vera, R. (2016, August 21). Why did young Filipinos vote for Bongbong Marcos? Lifestyle.inq. Retrieved from http://lifestyle.inquirer.net/235777/why-did-young-filipinos-vote-for-bongbong-marcos/

De Vera, B. (2018, July 30). Gov't debt to breach P8T in 2019. Retrieved from https://business.inquirer. net/254811/govt-debt-breach-p8t-2019.

Distribution of income or consumption by quintile. (2015). Retrieved from http://povertydata.worldbank. org/poverty/country/PHL

Dlodlo, A. (2011, September). Ayesab'amagwala and the heritage of uMkhonto weSizwe. ANC Today: Online Voice of the African National Congress, 11 (35), 16-22. Retrieved from http://www.anc.org.za/ docs/anctoday/2011/pdf/at35.pdf

Docena, H. (2006, March-June). People power and the perils of democracy lite. Retrieved from http://pcij. org/stories/people-power-and-the-perils-of-democracy-lite/.

Doronila, A. (2006, August 28). Time for paradigm shift. Philippine Daily Inquirer. p. A1.

Doyo, M. C. (2016, September 22). Martial law massacres. Philippine Daily Inquirer, p. A11 Retrieved from https://www.pressreader.com/philippines/philippine-daily-inquirer/20160922/281745563861343 
Ellao, J. A. (2015, February 26). Where were you during the Edsa People Power in 1986? Retrieved from https://www.bulatlat.com/2015/02/26/where-were-you-during-the-edsa-people-power-in-1986/

Ellao, J.A. (2014, September 11). From Marcos to Aquino, a picture of failed agrarian reform. Retrieved from https://www.bulatlat.com/2014/09/11/from-marcos-to-aquino-a-picture-of-failed-agrarianreform/

Engels, F. (1856, February 7). Engels to Marx in London. Retrieved from https://marxists.catbull.com/ archive/marx/works/1856/letters/56_02_07.htm (Original published in 1929)

Engels, F. (1885, May 15). Engels to Schlüter (L. E. Mins, Trans). Retrieved from http://www.marxists.org/ archive/marx/works/1885/letters/85_05_15.htm (Original published in 1938)

Escalante massacre. (2015, October 12). Retrieved from http://www.bantayog.org/escalante-massacre/

Espada, D. (2004, January 31). Remembering the Mendiola massacre: "Amid the assault, we were undaunted." Retrieved from https://www.bulatlat.com/2004/01/31/remembering-the-mendiolamassacre-amid-the-assault-we-were-undaunted/

Fagan, D. (2008). The left and the future of the Marcos Regime in the Philippines. Journal of Contemporary Asia, 5(2), 269-282. https://doi.org/10.1080/00472337508566950

Final results: 2016 presidential and vice presidential canvass. (2016, May 27). Retrieved from https:// newsinfo.inquirer.net/787867/philippine-elections-final-results-2016-congressional-canvasspresidential-vice-president-race\#ixzz5jGBijBFp

Floro, M. (2017, February 24). Yano: Icon of the People Power revolution. Retrieved from http://www. onemusic.ph/news/yano-icon-of-the-people-power-revolution-156

GetRealPost. (2016, February 7). Imperial Manila: Uniting the Nation at a Price. Retrieved from https:// www.getrealphilippines.com/2016/02/imperial-manila-uniting-nation-price/

Maceda, T. (1996). Mga tinig mula sa ibaba: kasaysayan ng partido komunista ng Pilipinas at partido sosialista ng Pilipinas sa awit, 1930-1955. Quezon City: University of the Philippines Press.

Gluckstein, D. (2008, October 6). Decyphering the internationale: the Eugène Pottier code. International Socialism: A Quarterly Journal of Socialist Theory, 120. Retrieved from http://www.isj.org.uk/index. php4?id $=485$

Gramsci, A. (1985). Problems of Criticism cultural writings (W. Boelhower,Trans.). In D. Forgacs and G. Nowell-Smith (Eds.), Selections from cultural writings. London: Lawrence and Wishart.

Gregory, M. (2010). Solidarity forever. Retrieved from http://unionsong.com/u025.html

Gržinić, M. (2011). Linking theory, politics, and art. In. Z. Kocur (Ed.), Global visual cultures: an anthology. West Sussex: Blackwell Publishing Ltd.

Guillermo, G. (1998).Muog:Ang naratibo ng kanayunan sa matagalang digmang bayan sa Pilipinas. Quezon City: University of the Philippines Press.

Hacienda Dolores: Testament to CARP failure. (2014, June 14). Retrieved from http://ibon.org/2014/06/ hacienda-dolores-testament-to-carp-failure/

Hawes, G. (1986, June). United States support for the Marcos administration and the pressures that made for change. Contemporary Southeast Asia 8(1), 18-36. Retrieved from https://www.jstor.org/ stable/25797880?seq=1\#page_scan_tab_contents

Hernandez, C.(1985.October).The Philippine military and civilian control:under Marcos and beyond.Third World Quarterly, 7(4), 907-923. Retrieved from https://www.jstor.org/stable/3991758?seq=3\#page_ scan_tab_contents 
Irfan Şenozan. (2017, July 23). Bella ciao English version [Video file]. Retrieved from https://www.youtube. com/watch?v=aOFjc1rUoB4

istvan piroth. (2015, January 26). Bella ciao Deutsch Italienisch hannes wader konstantin wecker [Video file]. Retrieved from https://www.youtube.com/watch?v=luEx_lq_7W8

Jiang, H. (2010). The Paris commune in Shanghai: The masses, the state, and dynamics of "continuous revolution." (Unpublished dissertation). Duke University. Retrieved from https://dukespace.lib.duke. edu/dspace/bitstream/handle/10161/2356/D_Jiang_Hongsheng_a_201005.pdf

Jimenez, F. (2008, December 23). Civil group member says sorry for helping put Arroyo in power. GMA News Online. Retrieved from http://www.gmanetwork.com/news/news/nation/140911/civilgroup-member-says-sorry-for-helping-put-arroyo-in-power/story/

Kerkvliet, B. (1974). Land reform in the Philippines since the arcos coup. Pacific Affairs, 47(3), 286-304. doi: $10.2307 / 2755767$

Kimuell-Gabriel, N. (2013). Ang kababaihan ng Tundo sa panahon ng batas militar. Scientia, 1(2), 10-43. Retrieved from http://scientia-sanbeda.org/vol-21/

KMP tells Congress to bury the long-dead CARP, legislate GARB. (2018, June 1). Retrieved from http:// davaotoday.com/main/politics/kmp-tells-congress-to-bury-the-long-dead-carp-legislate-garb/.

kodao phils. (2015, March 24). "Minsan karaniwang tao" - Chikoy Pura of The Jerks with Renato Reyes, Jr. of BAYAN. [Video file]. Retrieved from https://www.youtube.com/watch?v=87-HjJsDKDs

Kundera, M. (1996). Lost letters, the book of laughter and forgetting. London: Faber and Faber Limited.

Lapeña, C. (2012, January 19). Mendiola massacre: Survivor recounts horror 25 years later. GMA News Online. Retrieved from http://www.gmanetwork.com/news/news/specialreports/245040/ mendiola-massacre-survivor-recounts-horror-25-years-later/story/

Lieberman, R. (1995). My song is my weapon: people's songs, American communism, and the politics of culture, 1930-1950.USA:Illini Books.

Litonjua, M.D. (2001). The state in development theory:The Philippines under Marcos. Philippine Studies 49(3) (Third Quarter 2001), pp.368-398.

Llanes, R. (2013, March 15). Character actor-comedian Subas Herrero dies of pneumonia. Retrieved from https://www.pep.ph/news/37855/character-actor-comedian-subas-herrero-dies-of-pneumonia

Lolarga, E. (2002, June 1-15. Apo Hiking Society after 34 years: it's still a new day. Planet Philippines Online. Retrieved from https://web.archive.org/web/20080519070924/http://www.planetphilippines. com/archives/jun1-15/features_current/feature1.html

Lockard, C. (1998). Dance of life: Popular music and politics in Southeast Asia. University of Hawaii Press.

Lopez, V. (2016, August 22). Burying Marcos at Libingan ng mga Bayani not illegal, SolGen tells SC. Retrieved from https://www.gmanetwork.com/news/news/nation/578534/burying-marcos-atlibingan-ng-mga-bayani-not-illegal-solgen-tells-sc/story/

Luz, G. (2016, February 27). The 1986 Edsa revolution and the 2016 elections. Retrieved from https:// opinion.inquirer.net/93242/the-1986-edsa-revolution-and-the-2016-elections

Malalad, G. (2010, May 31). Apo takes a bow. ABS-CBN News Online. Retrieved from http://www.abscbnnews.com/entertainment/05/31/10/apo-takes-bow

Mann, K. (2011 July/August). Remembering the Paris commune. Retrieved from https://solidarity-us.org/ atc/153/p3315/ 
Marx, K. (1844, August 7 and 10). Critical notes on the article: "The king of Prussia and social reform" by a Prussian. Retrieved from http://www.marxists.org/archive/marx/works/1844/08/07.htm\#art2 (Original published in 1844)

Marx, K. (1871). The civil war in France. Retrieved from https:/www.marxists.org/archive/marx/ works/1871/civil-war-france/ch05.htm (Original published in 1871)

مالسلا ةراثيق نامع Moody. Published on Nov 17, 2018. (2018, November 17). Bella Ciao (Omani) يدووم [Video file]. Retrieved from https://www.youtube.com/watch?v=Gj4fMsW8FsQ

Mutasa, H. (2011, September 12). 'Shoot the boer' freedom song banned [blog post]. Retrieved from https://www.aljazeera.com/blogs/africa/2011/09/71901.html

Mydans, S. (1986). The Philippine middle class: Turning against Marcos. New York Times. Retrieved from https://www.nytimes.com/1986/02/02/magazine/the-philippine-middle-class-turning-againstmarcos.html

Mydans, S. (2006, February 20). Enthusiasm wanesfor people power. New York Times. Retrieved from https://www.nytimes.com/2006/02/20/world/asia/enthusiasm-wanesfor-people-power.html

Navarro, R. (2008, January-December). Ang bagong lipunan, 1972-1986: Isang panimulang pag-aaral sa musika at lipunan. Humanities Diliman, 5(1\&2), 47-77. Retrieved from http://journals.upd.edu.ph/ index.php/humanitiesdiliman/article/view/1480

Nemenzo, F. (1985). The left and the traditional opposition. Retrieved from http://lynchlibrary.pssc. org.ph:8081/bitstream/handle/0/4890/Nemenzo\%2C\%20Francisco.\%20The\%20Left $\% 20$ and\%20the\%20Traditional\%200pposition\%20in\%20The\%20Philippines\%20After\%20Marcos. pdf?sequence $=1$ \&isAllowed $=y$

Nuprokevin. (2017, July 23). 啊朋友 , 再见 ! 《桥》主题曲 Bella Ciao [Video file]. Retrieved from https:// www.youtube.com/watch?v=a79GGtuV-T8

Ocampo, S. (2011, March 5). Edsa 1, Broadly. Retrieved from https://www.bulatlat.com/2011/03/05/saturc-ocampo-edsa-1-broadly/

Ocampo, S. (2017, February 25). Different perspectives on the 1986 Edsa uprising. Retrieved from https:// www.philstar.com/opinion/2017/02/25/1674690/different-perspectives-1986-edsa-uprising

Olea, R. (2009, August 9). An Appraisal: Cory Aquino and Human Rights. Retrieved from https://www. bulatlat.com/2009/08/09/an-appraisal-cory-aquino-and-human-rights/

Olea, R. (2018, September 29). If Art is a Hammer: Pounding on Duterte's tyranny. Retrieved from https:// www.bulatlat.com/2018/09/19/art-hammer-pounding-dutertes-tyranny/

Orosa, R. (2009, November 12). APO show reset for Nov. 28. Philstar Global. Retrieved from https://www. philstar.com/entertainment/2009/11/12/522269/apo-show-reset-nov-28

Ortega, S. (1975). ¡El pueblo unido, jamás será vencido! [Recorded by Quilapayun]. On ${ }_{i} E l$ pueblo unido, jamás será vencido! [LP]. Germany: Pläne, Dicap.

Palatino, M. (2016, December 29).The political legacy of the Filipino sixty-eighters. Retrieved from https:// www.bulatlat.com/2016/12/29/political-legacy-filipino-sixty-eighters/

Paredes, J. (1986). Handog ng Pilipino sa mundo. [Recorded by Various Artists]. On Handog ng Pilipino sa Mundo [CD]. Quezon City:WEA.

Paredes, J. (2007, October 27). Equal right to lawlessness. Retrieved from http://jimparedes.com/archives/ date/2007/10

Paredes, J. (2016, February). How Jim Paredes gave people power its anthem, and why we'll never 
stop singing it. Rogue. Retrieved from http://rogue.ph/how-jim-paredes-gave-people-power-itsanthem-and-why-well-never-stop-singing-it/

Parsing Edsa. [Editorial]. (2019, February 25). Retrieved from https://www.sunstar.com.ph/ article/1794167.

Philippines' 50 richest (n.d.). Retrieved from www.forbes.com/philippines-billionaires/list

Philippines' poverty rate declines; More well-paying jobs and opportunities needed. (2018, May 30). Retrieved from https://www.worldbank.org/en/news/press-release/2018/05/30/philippinespoverty-rate-declines-more-well-paying-jobs-and-opportunities-needed

Poverty \& equity data portal. (n.d.). Retrieved from https://youtu.be/4dN5auAgTVQ

Pura, C. (1997). Sayaw sa bubog. [Recorded by The Jerks]. On The Jerks [CD]. Quezon City: Star Recording, Inc.

Remollino, A.M.(2006, February 19-25).Twenty people who made EDSA I possible. Bulatlat. Retrieved from https://www.tapatalk.com/groups/defense_philippines/20-people-who-made-edsa-i-possiblet1334.htmlhttp://www.bulatlat.com/news/6-3/6-3-people.htm

Revising the budget process in order to institutionalize the budgetary innovations of the new society (Presidential Decree No.1177). Retrieved from https://www.lawphil.net/statutes/presdecs/pd1977/ pd_1177_1977.html

Richburg, K. (1987, January 23). Manila troops kill 12 protesters. The Washington Post. Retrieved from https://www.washingtonpost.com/archive/politics/1987/01/23/manila-troops-kill-12protesters/5c1dd1a0-4531-4110-87ea-d8e9f94b7838/?utm_term=.1c4e3cdcbbd8

Rivera, T. (2001). The middle classes and democratization in the Philippines: from the Asian crisis to the ouster of Estrada. Retrieved from http://cenpeg.org/fellows_speak/rivera/Rivera\%20The\%20 Middle\%20Classes\%20and\%20Democratization.pdf

Roces, M. (2004). The militant nun as political activist and feminist in martial law Philippines. PORTAL Journal of Multidisciplinary International Studies, 1(1), 1-26. Retrieved from https://epress.lib.uts.edu. au/journals/index.php/portal/article/view/49

Romantic Lyrics. (2018, April 18). Bella ciao en español | Diego moreno (Lyrics/Paroles)version! [Video file]. Retrieved from https://www.youtube.com/watch?v=FGMvkIPUM18

San Juan, D. M. (2014). Pambansang salbabida at kadena ng dependensiya: Isang kritikal na pagsusuri sa Labor Export Policy (LEP) ng Pilipinas. Malay 27(1), 46-68. Retrieved from https://ejournals.ph/ article.php?id=8066

San Juan, D. M. (2016). Neoliberal restructuring of education in the Philippines: dependency, labor, privatization, critical pedagogy, and the K to 12 system. Asia-Pacific Social Science Review 16(1), 80-110. Retrieved from https://www.researchgate.net/publication/320558212_Neoliberal_ Restructuring_of_Education_in_the_Philippines_Dependency_Labor_Privatization_Critical_ Pedagogy_and_the_K_to_12_System

Santiago, J. (2008). Pagbabago. [Recorded by Jess Santiago] On Puso at Isip [CD]. Quezon City: Himigbayan Productions.

Slumbering Colossus. (2011, October 22). Pete Seeger and occupy wall street sing "We shall overcome" [Video file]. Retrieved from https://www.youtube.com/watch?v=-vgMu0_xKE0 
Severo, J.M. (2018, June 26). Stars react to Duterte's "stupid God" remark. Philstar Global. Retrieved from https://www.philstar.com/entertainment/2018/06/26/1828098/stars-react-dutertes-stupid-godremark\#5rbG49hVVGYoQTFv.99

Steinberg, D. J. (ed). (1987). In search of Southeast Asia: A modern history (revised). Hawaii: University of Hawaii Press.

Sulat, B. (2013, March 15). Subas Herrero of "Champoy" dies. Retrieved from https://www.rappler.com/ entertainment/23892-tv-movie-actor-subas-herrero-dies

Tadem, E. (2015, September 15). Marcoses should apologize to Filipino farmers. Inquirer.net. Retrieved from https://opinion.inquirer.net/88150/marcoses-should-apologize-to-filipino-farmers

Tan, K.J. (2009, August 6). Top singers turn mass for Cory into mini-concert. Retrieved from https://www. gmanetwork.com/news/showbiz/content/169185/top-singers-turn-mass-for-cory-into-miniconcert/story/

Tensions erupt between Duterte Youth and Jim Paredes at EDSA 31 celebration. (2017, February 26). Retrieved from http://nine.cnnphilippines.com/news/2017/02/26/Jim-Paredes-Duterte-youthEDSA-31-confrontation.html

The Serbian Partisan. (2018, October 5). Çav bella - Turkish Bella Ciao with English lyrics [Video file]. Retrieved from https://www.youtube.com/watch?v=R3F0T1aJzMI

Thousands join million people march vs pork. (2013, August 26). Retrieved from http://news.abs-cbn. com/nation/08/26/13/thousands-join-million-people-march-vs-pork

Thompson, M. \& C. Ramhurry. (2014). A uniting song that divides: A critical analysis of Aw Dubul'ibhunu (Kill the Boer).Muziki, 11(1), 32-42. doi: https://doi.org/10.1080/18125980.2014.893091

Tiglao, R. (2015, September 20). Why did the military support the Marcos dictatorship?. Manila Times. Retrieved from http://www.manilatimes.net/why-did-the-military-support-the-marcosdictatorship/219754/

Tuazon, B. (2004, November 21-24). The Hacienda Luisita massacre, landlordism and state terrorism. Retrieved from https://www.bulatlat.com/2004/11/21/the-hacienda-luisita-massacre-landlordismand-state-terrorism/

Tubeza, P. (2001, February 13). Lagman buried at sunset. Retrieved from http://popoylagman.blogspot. com/2009/02/lagman-buried-at-sunset.html

Tuovalla. (2007, November 19). The internationale in 47 languages +1 instrumental version! [Video file]. Retrieved from https://www.youtube.com/watch?v=m7PrVtZAG4M

TV5 InterAksyon. (2016, September 20). Mga awit ng pagkamulat: "Saranggola ni Pepe" By Nonoy Gallardo. [Video file] Retrieved from https://www.youtube.com/watch?v=tmQDt5HluKg.

Employment situation in January 2018 (final results). (2018, June 14). Retrieved from https://www.psa. gov.ph/content/employment-situation-january-2018-final-results

Valeros, M.E. (2006, November 10). Dongabay moves on with "Flipino." Philippine Star. Retrieved from https://www.philstar.com/cebu-entertainment/2006/11/10/368081/dongabay-moves-flipino

Velasco, D. (2004). Kompil II: A Study of Civil Society's Political Engagements. Philippine Sociological Review 52 (January-December 2004), 97-118.

Walang Noranian na naging drug addict dahil kay Nora: Fans rail vs PNoy's reason for National Artist snub. (2014, July 1). Retrieved from https://www.gmanetwork.com/news/showbiz/content/368139/ walang-noranian-na-naging-drug-addict-dahil-kay-nora-fans-rail-vs-pnoy-s-reason-for-national- 
artist-snub/story/Wurfel, D. (1977). Martial law in the Philippines: The methods of regime survival. Pacific Affairs, 50(1), 5-30. doi: 10.2307/2756116

Youngblood, R. (1978). Church opposition to martial law in the Philippines. Asian Survey, 18(5), 505-520. doi: $10.2307 / 2643463$

Ylagan, A. (2014, May 5). A contented middle class. Business World. Retrieved from http://www. bworldonline.com/content.php?section=Opinion\&title=a-contented-middle-class\&id=86944

Ylagan, A. (2018, February 25). The people power that was. BusinessWorld. Retrieved from https://www. bworldonline.com/the-people-power-that-was/

Zinn, H. at A. Arnove. (2004). Voices of a people's history of the United States. USA: Seven Stories Press.

DAVID MICHAEL M. SAN JUAN is an associate professor at the Filipino Department of De La Salle University-Manila, convenor ng Alyansa ng Mga Tagapagtanggol ng Wikang Filipino (TANGGOL WIKA), president of Pambansang Samahan sa Linggwistika at Literaturang Filipino (PSLLF), and vice head of the National Committee on Language and Translation under the National Commission for Culture and the Arts. (Corresponding author:david.sanjuan@dlsu.edu.ph ) 
